\title{
Peripheral calcium-permeable AMPA receptors regulate chronic inflammatory pain in mice
}

\author{
Vijayan Gangadharan, ${ }^{1}$ Rui Wang, ${ }^{2}$ Bettina Ulzhöfer, ${ }^{1}$ Ceng Luo, ${ }^{1}$ Rita Bardoni, ${ }^{3}$ \\ Kiran Kumar Bali,1 Nitin Agarwal,1 Irmgard Tegeder, ${ }^{4}$ Ullrich Hildebrandt, ${ }^{1}$ \\ Gergely G. Nagy, ${ }^{5,6}$ Andrew J. Todd, ${ }^{5}$ Alessia Ghirri, ${ }^{3}$ Annette Häussler, ${ }^{4}$ Rolf Sprengel, ${ }^{7}$ \\ Peter H. Seeburg, ${ }^{7}$ Amy B. MacDermott, ${ }^{8}$ Gary R. Lewin, ${ }^{2}$ and Rohini Kuner ${ }^{1}$

\begin{abstract}
${ }^{1}$ Institute for Pharmacology, Heidelberg University, Heidelberg, Germany. ${ }^{2}$ Max Delbrück Center for Molecular Medicine, Berlin, Germany. 3Department of Biomedical Sciences - Physiology, University of Modena and Reggio Emilia, Modena, Italy. ${ }^{4}$ Pharmazentrum Frankfurt, Klinikum der Johann Wolfgang Goethe-Universität, Frankfurt am Main, Germany. ${ }^{5}$ Spinal Cord Group, West Medical Building, University of Glasgow, Glasgow, United Kingdom. ${ }^{6}$ First Department of Internal Medicine, University of Debrecen,

Medical and Health Science Center, Debrecen, Hungary. 'Department of Neurobiology, Max Planck Institute for Medical Research, Heidelberg, Germany. ${ }^{8}$ Department of Physiology and Cellular Biophysics, Columbia University, New York, New York, USA.
\end{abstract}

\begin{abstract}
a-Amino-3-hydroxy-5-methyl-4-isoxazolepropionic acid-type (AMPA-type) glutamate receptors (AMPARs) play an important role in plasticity at central synapses. Although there is anatomical evidence for AMPAR expression in the peripheral nervous system, the functional role of such receptors in vivo is not clear. To address this issue, we generated mice specifically lacking either of the key AMPAR subunits, GluA1 or GluA2, in peripheral, pain-sensing neurons (nociceptors), while preserving expression of these subunits in the central nervous system. Nociceptor-specific deletion of GluA1 led to disruption of calcium permeability and reduced capsaicin-evoked activation of nociceptors. Deletion of GluA1, but not GluA2, led to reduced mechanical hypersensitivity and sensitization in models of chronic inflammatory pain and arthritis. Further analysis revealed that GluA1-containing AMPARs regulated the responses of nociceptors to painful stimuli in inflamed tissues and controlled the excitatory drive from the periphery into the spinal cord. Consequently, peripherally applied AMPAR antagonists alleviated inflammatory pain by specifically blocking calcium-permeable AMPARs, without affecting physiological pain or eliciting central side effects. These findings indicate an important pathophysiological role for calcium-permeable AMPARs in nociceptors and may have therapeutic implications for the treatment chronic inflammatory pain states.
\end{abstract}

\section{Introduction}

Chronic pain represents a frequent and poorly understood medical problem. Plasticity of synaptic transmission in the nervous system following inflammation in peripheral organs or nerve injury is an important component of the cellular basis of chronic, pathological pain (1). Glutamate serves as the primary excitatory neurotransmitter at several key synapses in the somatosensory nociceptive pathway, in which it activates both ionotropic and metabotropic receptors. Recently, the $\alpha$-amino-3-hydroxy-5-methyl-4-isoxazolepropionic acid-type (AMPA-type) glutamate receptors (AMPARs) have emerged as important mediators of synaptic plasticity in the brain $(2,3)$. In contrast to NMDA-type glutamate receptors, which always permit $\mathrm{Ca}^{2+}$ entry upon activation, AMPARs constitute an activity-dependent switch, which controls glutamate-evoked entry of $\mathrm{Ca}^{2+}$ into neurons (4). This activity-dependent switch is brought about by regulated expression and inclusion of the subunit GluA2 (ref. 5; previously called GluR-B or GluR2), which imparts low $\mathrm{Ca}^{2+}$ permeability to AMPAR channels $(3,4)$. In contrast, the GluA1 subunit (previously called GluR-A or GluR1) is highly expressed in regions that demonstrate a high density of calcium-permeable

Authorship note: Vijayan Gangadharan and Rui Wang contributed equally to this work.

Conflict of interest: The authors have declared that no conflict of interest exists. Citation for this article: J Clin Invest. 2011;121(4):1608-1623. doi:10.1172/JCI44911.
AMPARs, including components of pain pathways (5-7). Although our previous studies in global gene knockouts of AMPAR subunits revealed an important role for GluA1-containing AMPARs in chronic pain mechanisms (6), they did not identify the anatomical venue involved. Indeed, AMPARs are expressed across several key modulatory regions in the somatosensory pathways mediating pain, such as peripheral nociceptive neurons, spinal dorsal horn, ventral horn, and in several brain regions (5-8), which govern the sensory, affective, and emotional components of pain. However, the differential, relative contributions of these regions toward central sensitization and chronic pain have so far not been elucidated.

All peripheral sensory neurons use glutamate as their primary transmitter, and major subsets of sensory neurons of the dorsal root ganglia (DRG) are known to express mRNA or show immunoreactivity for ionotropic and metabotropic glutamate receptors. Electron microscopy studies provided compelling evidence that AMPAR subunits are transported into peripheral processes of sensory neurons (e.g., ref. 8), and recent anatomical and ex vivo electrophysiological data have also suggested presynaptic localization and functional involvement of AMPAR subunits in spinal terminals $(9,10)$. However, the functional roles of AMPARs situated on central and peripheral terminals of sensory neurons in nociceptive modulation in vivo, at the level of the whole organism, have remained unclear. Furthermore, because AMPARs are also expressed in peripheral sympathetic neurons, Schwann cells, and 
keratinocytes (11), the use of pharmacological agents alone does not fully permit the dissection of AMPAR contribution at different loci in the modulation of pain in vivo.

Here, we have used the Cre-loxP system (12) to generate transgenic mice that selectively lack the key AMPAR subunits, GluA1 or GluA2, specifically in the peripheral arm of the somatosensory pain pathway, i.e., in the soma, axons, and the peripheral and central terminals of nociceptive neurons of the DRG, leaving the AMPAR in the central nervous system intact. A combination of electrophysiologic, pharmacologic, and genetic approaches revealed that GluA1-containing, GluA2-lacking calcium-permeable AMPARs modulate responses of peripheral nociceptors to algogens. Furthermore, we observed that calcium-permeable AMPARs play distinct roles in physiological (i.e., noninjured) and postinflammatory states. In contrast, GluA2-containing calcium-impermeable AMPARs, which can be heteromers of GluA2 with GluA1, GluA3, or GluA4, in nociceptors were found to be dispensable for pain modulation. Our results demonstrate that GluA1-containing AMPARs expressed in peripheral nociceptive neurons are important modulators of peripheral sensitization as well as peripheral mechanisms triggering central hyperexcitability in inflammatory states and represent what we believe to be novel targets for pain therapeutics.

\section{Results}

SNS-Cre-mediated conditional deletion of GluA1 or GluA2 specifically in peripheral nociceptive neurons. We have previously reported that a mouse line expressing the Cre recombinase under control of the $\mathrm{Na}_{\mathrm{v}} 1.8$ promoter (SNS-Cre) enables conditional gene deletion specifically in peripheral nociceptive neurons in the DRG, while leaving the expression in central nervous system and nonneural cells intact (e.g., refs. 13, 14). Accordingly, we generated mice lacking GluA1 or GluA2 specifically in peripheral nociceptive neurons (SNS-GluA1-/- and SNS-GluA2-/- mice, respectively) via Cre/loxPmediated recombination by mating SNS-Cre mice with mice carrying the floxed Gria1 allele (GluA1 flfl; ref. 15) or Gria2 allele $\left(G \operatorname{luA} 2^{f l / f l}\right.$; ref. 16) (Figure 1, A and B). An anti-GluA1 antibody showed widespread immunoreactivity in small-diameter as well as large-diameter neurons of adult mouse DRG, consistent with a previous report on neonatal rat DRG (9), but showed a complete lack of staining in DRG, spinal cords, and brains derived from classical (global) deletion mutants of GluA1, thereby demonstrating its specificity (Figure 1A). Using this antibody, we found that only large-diameter neurons of the DRG in SNS-GluA1-/- mice, which mostly represent somata of tactile afferents and proprioceptors, continued to show GluA1 expression. In contrast, anti-GluA1 immunoreactivity was markedly reduced in small-diameter neurons, a majority of which constitute somata of C- and A- $\delta$ nociceptors (typical examples in Figure 1A and size-frequency analysis in Figure 1C). This was supported by a specific expression of the Cre recombinase in small-sized neurons of the DRG in SNS-GluA1 $1^{-/-}$ mice, as judged via anti-Cre immunoreactivity (Figure 1A). Dual immunohistochemistry using anti-GluA1 and anti-calcitonin gene-related peptide (CGRP) antibodies (markers of peptidergic nociceptive neurons) or isolectin- $\mathrm{B}_{4}$ ( $\mathrm{IB}_{4}$; a marker for nonpeptidergic nociceptors) indicated a selective deletion of anti-GluA1 immunoreactivity in nociceptors of SNS-GluA1 $1^{-/}$mice, whereas large-diameter neurons negative for CGRP expression/ $\mathrm{IB}_{4}$ binding retained expression of GluA1 (a quantitative summary is shown in Figure 1E and typical examples are shown in Supplemental
Figure 1; supplemental material available online with this article; doi:10.1172/JCI44911DS1). In contrast, GluA1 expression was preserved in the spinal cords and brains of SNS-GluA1-/- mice, verifying specificity of deletion in the DRG (Figure 1A and data not shown). Western Blot analysis on DRG, spinal cord, and brains of GluA1 $1^{f / f l}$ mice and SNS-GluA1 $1^{-/-}$mice further confirmed a DRGspecific loss of GluA1 in SNS-GluA1-/- mice (Figure 1F). GluA1 expression in the DRG was unchanged in SNS-GluA2 $2^{-/-}$mice (Supplemental Figure 2C).

SNS-GluA2 $2^{-/}$mice showed a similar profile of Cre-mediated gene deletion of GluA2 (Figure 1B). An antibody that has been widely used to characterize GluA2 expression (but which recognizes mouse GluA2 and GluA3) showed that anti-GluA2/3 immunoreactivity was markedly reduced, but not lost, in small-diameter DRG neurons in SNS-GluA2-/- mice (typical examples are shown in Figure 1B and mean summary of size frequency analysis is shown in Figure 1D). A newly described commercial antibody, which recognizes recombinant GluA2, but not GluA3, revealed a loss of GluA2 in CGRP-positive or $\mathrm{IB}_{4}$-positive neurons of SNSGluA2 $2^{-/-}$mice (Supplemental Figure 2, A and B) but not in those of SNS-GluA1 $1^{-/-}$mice (Figure 1E and Supplemental Figure 1). Furthermore, Western blot analysis with the same antibody yielded a band of the size expected for GluA2 in the DRG of GluA2fl/fl mice but not in SNS-GluA2-/- mice (Figure 1F). In contrast, the GluA2 band was retained in the DRG of SNS-GluA1-/- mice (Supplemental Figure 2C). Finally, DRG derived from SNS-GluA1-/- mice and SNS-GluA2 $2^{-/-}$mice did not show any changes in the expression of GluA2 and GluA1, respectively, ruling out compensatory changes in the expression of other GluR subunits upon conditional deletion of GluA1 or GluA2 (Figure 1, A and B).

Normal development of DRG neurons and theirperipheral and central afferent connections in SNS-GluA1-/- and SNS-GluA2-/- mice. SNS-Cre-mediated gene deletion in the DRG commences perinatally (E17.5-P0) (13), thus side-stepping potential embryonic developmental defects in early outgrowth and patterning of DRG afferents, which typically take place over E12.5-E14.5 in mice $(17,18)$. Nevertheless, we ascertained that SNS-GluA1 $1^{-/}$mice and SNS-GluA2-/- mice do not show any obvious developmental defects in the spinal sensory-motor circuitry. The distribution of peptidergic (CGRP-expressing) and nonpeptidergic ( $\mathrm{IB}_{4}$-binding) small-diameter neurons in the DRG and the pattern of their termination in the spinal dorsal horn was similar in adult SNS-GluA1 $1^{-/}$and SNS-GluA2-/- mice, as compared with that in $G l u A 1^{f l / f l}$ and $G l u A 2^{f / f l}$ mice, respectively (Supplemental Figure 3A). Similarly, innervation of peripheral organs, such as the glabrous skin of the hind paw, was normal in these conditional mouse mutants, as revealed by immunohistochemistry for PGP9.5, a marker of peripheral nerves, and CGRP (Supplemental Figure 3B). Finally, high-resolution microscopy revealed normal density of synapses between peptidergic (CGRP-expressing) and nonpeptidergic ( $\mathrm{IB}_{4}$-binding) nociceptive afferents and PSD-95-positive puncta (representing postsynaptic aspects of glutamatergic synapses) in

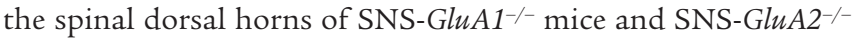
mice (Supplemental Figure 3C and see Supplemental Table 1 for quantification). We therefore concluded that SNS-GluA1-/- mice and SNS-GluA2 $2^{-/-}$mice show normal development of spinal and peripheral sensory circuits.

Presynaptic inhibitory effects of AMPA at spinal synapses. To directly test the presence and function of AMPARs on central terminals of primary afferent fibers, we compared sensitivity of transmitter release to exogenous AMPA $(120-250 \mathrm{nM})$ application in spinal 
A

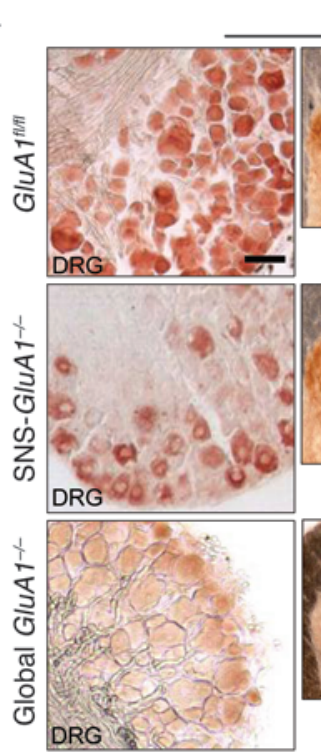

Anti-GluA1

Anti-Cre

Anti-GluA2/3

B
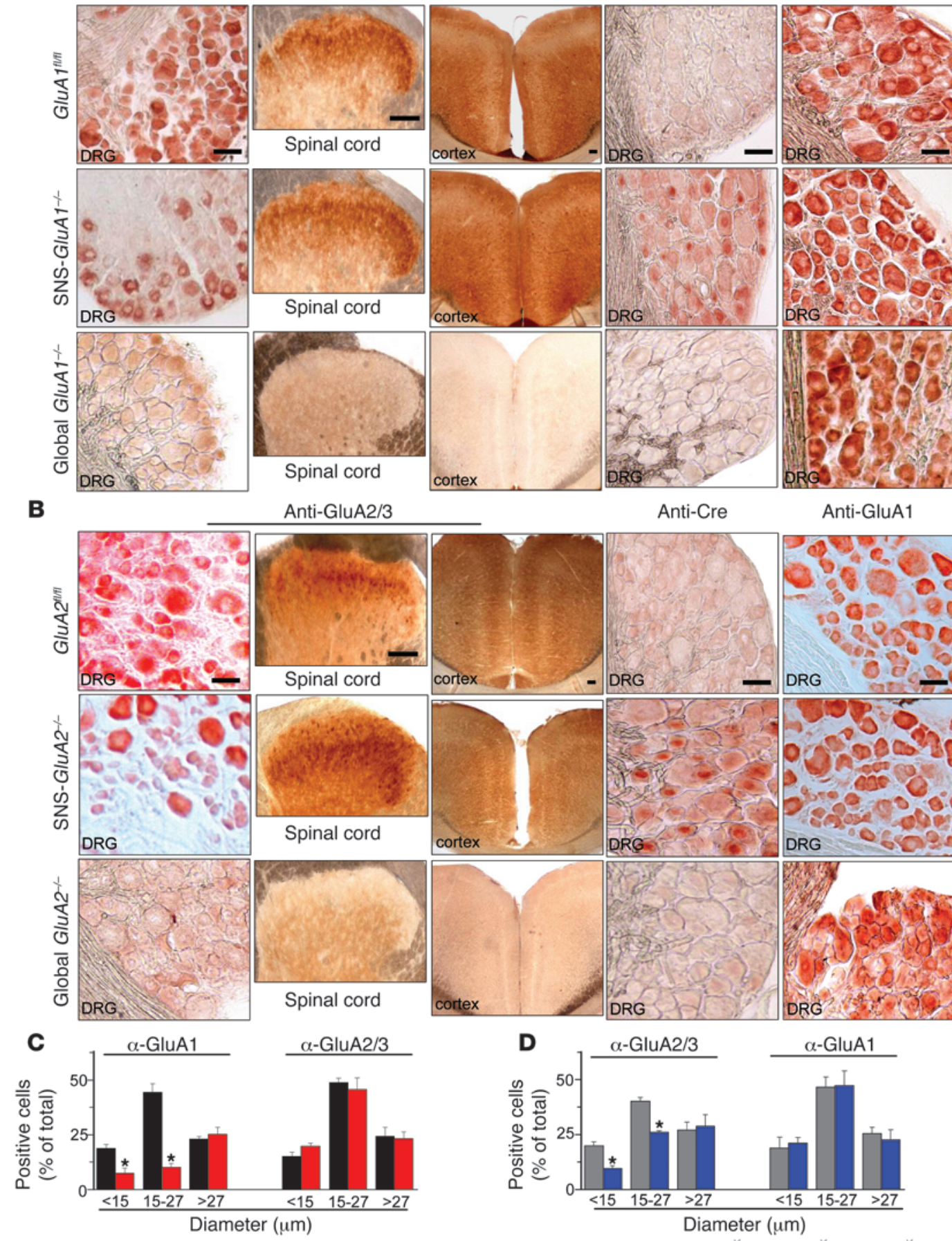

Spinal cord
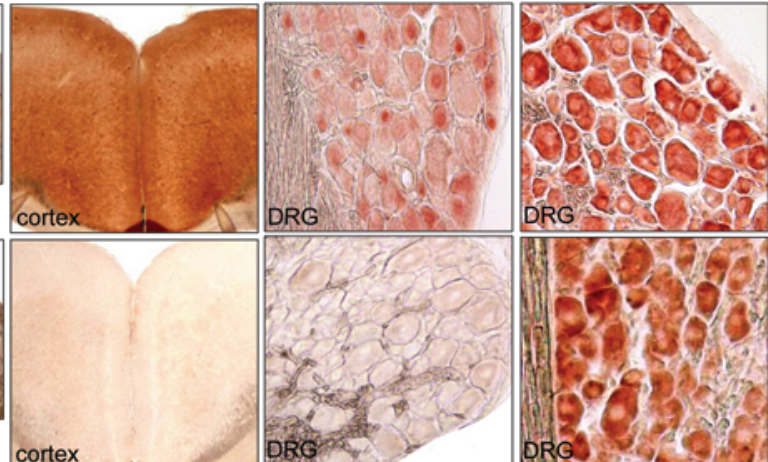

Anti-Cre

Anti-GluA1
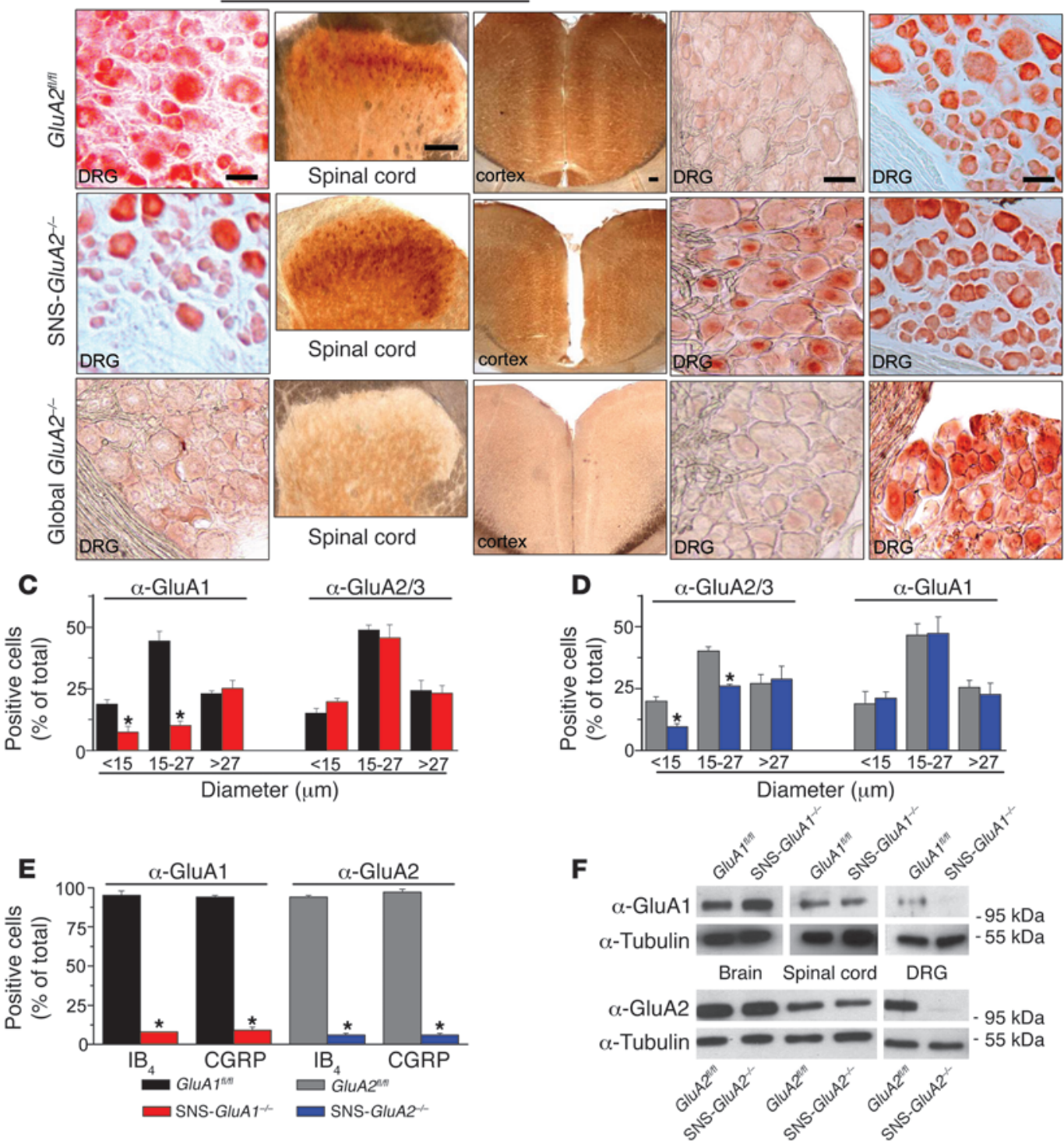


\section{Figure 1}

Generation and characterization of mice lacking GluA1 or GluA2 in Nav1.8-expressing neurons (nociceptors) of the DRG. (A) Immunohistochemistry with anti-GluA1, anti-Cre, and anti-GluA2/3 antibodies on sections of the DRG, spinal dorsal horn, and forebrain (anterior cingulate cortex is shown) of control mice (GluA $1^{f / f l}$ mice), nociceptor-specific GluA1 knockout mice (SNS-GluA1--- mice), and mice globally lacking GluA1 (GluA1-l- mice). (B) Immunohistochemistry with an antibody recognizing GluA2 and GluA3 (anti-GluA2/3) and anti-GluA1 and anti-Cre antibodies on sections of the DRG, spinal dorsal horn, and forebrain of control mice (GluA2 $2^{f / / f l}$ mice), nociceptorspecific GluA2 knockout mice (SNS-GluA2-- mice), and mice globally lacking GluA2 (GluA2-/- mice). (A and B) Scale bars: $50 \mu \mathrm{m}$ (DRG images); $100 \mu \mathrm{m}$ (spinal cord and brain images). (C and D) Quantitative size frequency analysis of DRG neurons immunoreactive against anti-GluA1 or anti-GluA2/GluA3 confirms that small-diameter neurons lose and large-diameter neurons maintain expression of the respective genetically targeted subunits in (C) SNS-GluA1-l- mice and (D) SNS-GluA2-/- mice. (C) In contrast, anti-GluA2/GluA3 immunoreactivity is maintained in SNS-GluA1/- mice, and (D) anti-GluA1 is maintained in SNS-GluA2 ${ }^{-/-}$mice. (E) Dual immunofluorescence quantitative analysis of anti-GluA1 and anti-GluA2 with markers of peptidergic nociceptors (CGRP) and nonpeptidergic nociceptors (IB 4 ) confirms a near complete loss of GluA1 in nociceptors of SNS-GIUA1-/- mice and loss of anti-GluA2 in SNS-GluA2-/- mice. In C-E, $y$ axes represent immunopositive cells represented as a percentage of all cells counted in corresponding DRG sections. ${ }^{*} P<0.05$ as compared to corresponding flox control mice. (F) Western blot analyses confirm DRG-specific deletion of GluA1 or GluA2 in SNS-GluA1-1- and SNSGluA2 ${ }^{-/-}$mice, respectively. Units for numbers in $F$ are $\mathrm{kDa}$. $\alpha$-Tubulin represents a loading control.

slices, as described by Lee et al. (9). Patch-clamp recordings in voltage-clamp mode were performed from a total of 43 lamina I and II neurons in slices derived from SNS-GluA1-/- mice, SNSGluA2-1- mice, and their respective control littermates. Glutamatergic monosynaptic excitatory postsynaptic currents (EPSCs) were evoked by dorsal root stimulation. In slices derived from all mouse populations, the application of AMPA consistently evoked a sustained increase in holding current, reflecting activation of postsynaptic AMPAR on the cell under study. Furthermore, EPSC peak amplitudes were significantly depressed by AMPA, with the percentage of inhibition varying among cells. Both effects of AMPA were rapidly reversible upon washout (Figure 2A). AMPAinduced EPSC inhibition has been reported to represent a sum of both postsynaptic and presynaptic actions of AMPA (9). To assess presynaptic AMPAR activity in the different populations, we analyzed changes in the coefficient of variation (CV) of EPSC amplitudes with AMPA application (19).

We constructed plots of the ratio of $\mathrm{CV}^{-2}\left(\mathrm{CV}^{-2} \mathrm{AMPA} / \mathrm{CV}^{-2}\right.$ control $)$ as a function of the relative EPSC amplitude $\left(\mathrm{EPSC}_{\mathrm{AMPA}} / \mathrm{EPSC}_{\mathrm{control}}\right)$, where $\mathrm{CV}^{-2}$ AMPA represents 1 /coefficient of variation squared, measured during application of AMPA; $\mathrm{CV}^{-2}$ control represents $1 /$ coefficient of variation squared, obtained in control; and $\mathrm{EPSC}_{\mathrm{AMPA}}$ and EPSC $_{\text {control }}$ represent mean amplitude of EPSCs, measured during AMPA application and in control, respectively, assuming that postsynaptic changes produce a change in mean amplitude without affecting the $\mathrm{CV}$ values, whereas presynaptic modulation would be expected to alter both parameters (20). As a control for this analysis, we recorded from lamina II neurons under control bath conditions, but at 2 different holding potentials ( -85 and $-55 \mathrm{mV}$ ), to mimic a pure postsynaptic modulation. Mean amplitude decreased at $-55 \mathrm{mV}$, while the $\mathrm{CV}$ value did not change (Figure 2B, $n=6)$. Conversely, lowering the concentration of extracellular calcium from $2 \mathrm{mM}$ to $1 \mathrm{mM}$ caused both a decrease of mean amplitude and a change of CV (Figure 2B, $n=7$ ), as would be expected for a presynaptic modulation.

Using these analysis parameters, we then compared data from mutant mice with their respective littermate controls. In the GluA1 fl/fl control group, 9 out of 10 neurons exhibited a presynaptic modulation of glutamate release. In contrast, depression of EPSC amplitude was not accompanied by a corresponding change of CV in 5 out of 12 cells recorded from SNS-GluA1-/- mice, suggesting involvement of a pure postsynaptic mechanism due to loss of presynaptic inhibition (Figure 2C). The mean ratio between CV (AMPA) and CV (control) was significantly lower in SNS-GluA1-/- mice as compared with that in GluA1 $1^{f l f l}$ mice (Figure 2E), indicating that presynaptic modulation by AMPA was less effective in spinal slices derived from SNS-GluA1-/- mice. In contrast, all neurons recorded from GluA2 $2^{f / f l}$ mice or from SNS-GluA2-/- mice exhibited changes of both $\mathrm{CV}$ and mean amplitude, suggesting the presence of presynaptic modulation (Figure 2D; $n=10$ and 11, respectively). Furthermore, the mean ratio between CV (AMPA) and CV (control) was similar in SNS-GluA2 $2^{-/}$mice and GluA2 $2^{f / f l}$ mice (Figure 2E). Taken together, these results suggest that GluA1-containing AMPARs are expressed near sites of transmitter release in primary sensory neurons and are involved in AMPA-induced depression of release from primary afferent nerve terminals in the spinal dorsal horn.

Modulation of peripheral afferent responses to heat and capsaicin by GluA1. We then addressed how the loss of GluA1-containing AMPARs affects the peripheral functions of nociceptors in target organs, such as the skin. Using the skin-nerve preparation (21), we recorded the activation of identified nociceptors to noxious stimuli, such as heat or capsaicin (Figure 3, A and B). The proportion of C-mechanonociceptors and C-mechanoheat fibers (C-MHs) excited by $100 \mu \mathrm{M}$ capsaicin was similar between SNS-GluA1-1and GluA1flfl mice (Figure 3F). Importantly, however, capsaicininduced spiking in nociceptors was significantly reduced in SNSGluA1 $1^{-/}$mice compared with that in GluA1 $1^{f / f l}$ mice (Figure 3, E and

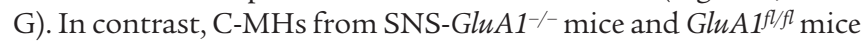
showed identical response characteristics to heat ramps applied to the receptive field. Thus, using a ramp and hold heat stimulus (ramp from $32^{\circ} \mathrm{C}$ to $52^{\circ} \mathrm{C}$ in 20 seconds hold at $52^{\circ} \mathrm{C}$ for $5 \mathrm{sec}-$ onds then ramp back to $32^{\circ} \mathrm{C}$ in 20 seconds), C-MHs displayed the same mean threshold temperature for the first and second spike (Figure 3, C and D). These data demonstrate that the magnitude of capsaicin-evoked excitation is reduced in C-nociceptors that lack GluA1-containing AMPARs; however, there is no change in sensitivity to noxious heat.

Analysis of the contribution of GluA1 to basal excitability in nociceptors. Using the whole cell mode of the patch clamp technique, we then analyzed the membrane properties of isolated DRG neurons with the physiological and morphological properties of nociceptors (small cell size and humped action potential; refs. 21, 22). The resting membrane potential of DRG neurons measured from SNSGluA1-/- and GluA1flffl mice were not different (Figure 4A). We then used step current injections to measure the threshold current for initiating action potentials but found no difference between the 2 genotypes (Figure 4B). We went on to use a ramp current injection to measure the latency for the first spike as well as the input-output curve (number of spikes against injection current); none of these parameters differed between neurons isolated from 
A

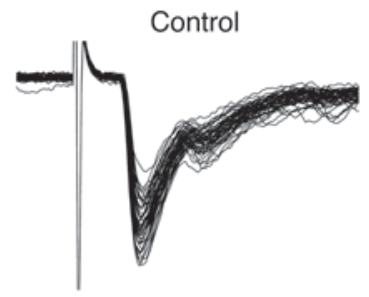

B

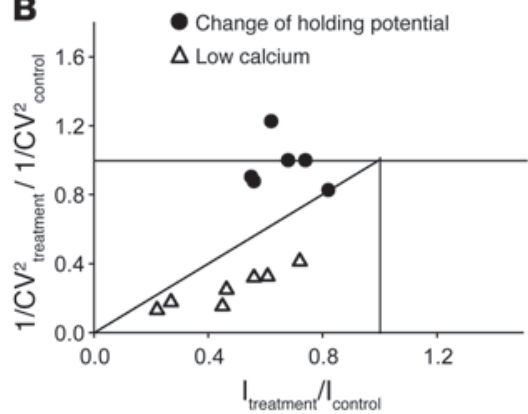

D

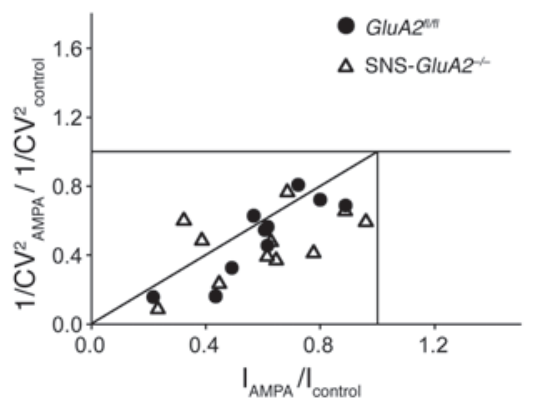

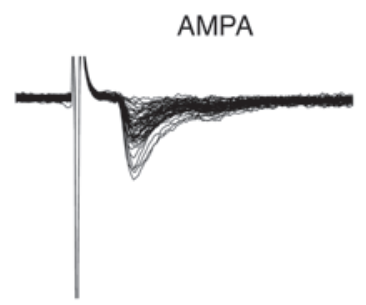

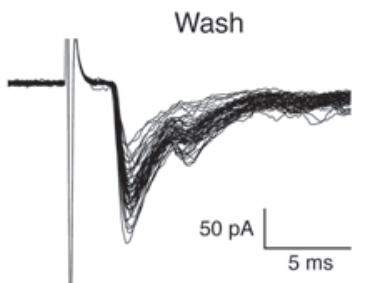

C

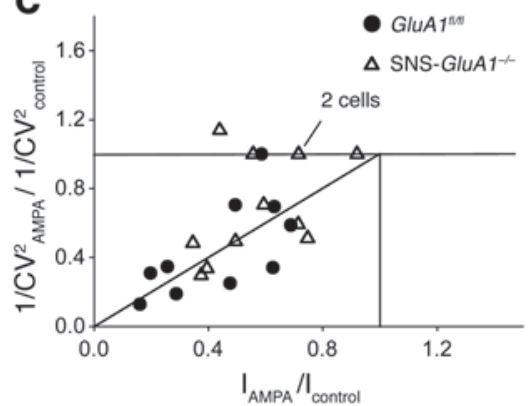

$\mathbf{E}$

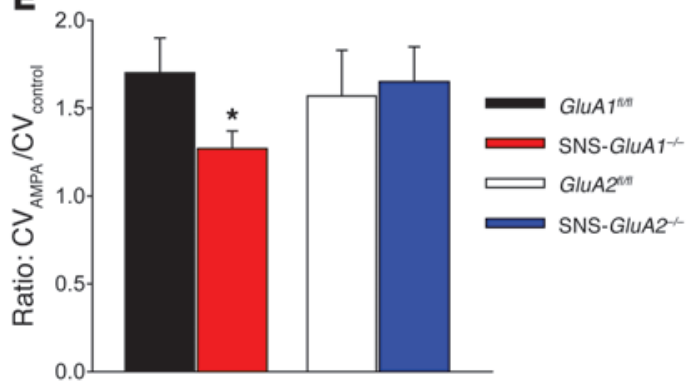

Figure 2

Patch clamp analysis of AMPA-induced modulation of synaptic transmission between primary afferents and spinal dorsal horn neurons in SNS-

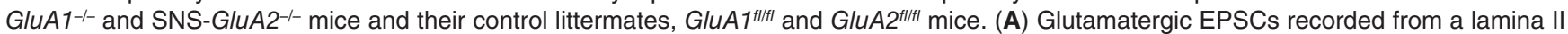
neuron obtained from a wild-type mouse. Application of AMPA (250 nM) caused a depression of EPSC, an increase of amplitude variability, and the appearance of some failures, which were reversed upon washing AMPA out. (B-D) Plot data showing the ratio of CV-2 as a function of relative EPSC amplitude. Each symbol represents 1 neuron. (B) Graph representing CV changes in control experiments (low extracellular calcium and change of holding potential) mimicking presynaptic and postsynaptic modulations, respectively. $1 / \mathrm{CV}^{2}{ }_{\text {treatment }} / 1 / \mathrm{CV}^{2}$ control represents the ratio between $1 /$ coefficient of variation squared, obtained during treatment (either $1 \mathrm{mM}$ extracellular calcium or holding potential $=-55 \mathrm{mV}$ ), and $1 / \mathrm{coefficient}$ of variation squared, measured in control (i.e., $2 \mathrm{mM}$ extracellular calcium or holding potential $=-85 \mathrm{mV}$ ). I treatment $/ \mathrm{l}_{\text {control }}$ represents the ratio between mean EPSC amplitude, measured during treatment (see above), and mean EPSC amplitude measured in control. (C and D) Effects of AMPA on

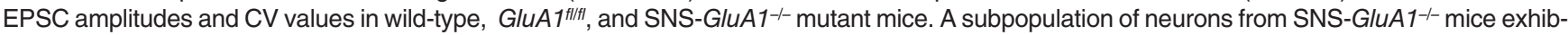
ited a pure postsynaptic modulation, since the $\mathrm{CV}$ remained constant in AMPA. $1 / \mathrm{CV}^{2}{ }_{\mathrm{AMPA}} / 1 / \mathrm{CV}^{2}{ }_{\text {control }}$ represents the ratio between $1 / \mathrm{coefficient}$ of variation squared, obtained during application of AMPA250 nM and 1/coefficient of variation squared, measured in control. $\mathrm{I}_{\text {AMPA }} / \mathrm{I}_{\text {control }}$ represents the ratio between mean EPSC amplitude, measured during AMPA application, and mean EPSC amplitude measured in control. (E) The ratio of CV in AMPA to CV in control is significantly lower in SNS-GluA1-l- mice, as compared with that in GluA1 $1^{t / f t}$ mice ${ }^{*} P<0.05, t$ test).

SNS-GluA1 $1^{--}$and GluA1flffl mice (Figure 4, C-E). We also found no differences in the magnitude of macroscopic inward and outward currents measured in voltage-clamp mode between neurons taken from SNS-GluA1-/- and GluA1 $1^{f l / f l}$ mice (data not shown). Thus, a loss of GluA1-containing AMPARs does not elicit a general change in the excitability of nociceptive neurons.

Calcium permeability of AMPARs in nociceptive neurons. The density of calcium-permeable AMPARs over most areas of the central nervous system is known to be low; moderate to high densities are only seen in specific areas and particular cell types (3-6). To address whether AMPARs expressed on peripheral nociceptive neurons are calcium permeable, we performed Fura-2-based calcium imaging on cultured DRG neurons derived from GluA1fl/fl and SNS-GluA1-/mice. Because SNS-Cre-mediated target gene deletion only occurs in $\mathrm{Na}_{\mathrm{v}} 1.8$-expressing nociceptors, there was a need for live identification of nociceptive neurons in this heterogenous mix of DRG neurons. We therefore used Alexa Fluor 488-conjugated $\mathrm{IB}_{4}\left(\mathrm{IB}_{4}-\right.$ Fluor488) for live identification of nonpeptidergic small-diameter nociceptive neurons, which constitute approximately $50 \%$ of nociceptors (Figure 4F; neurons dually labeled with Fura-2 and $\mathrm{IB}_{4}$-Fluor 488 are indicated by arrowheads); a method for live labeling of peptidergic neurons specifically is not available. Application of AMPA $(10 \mu \mathrm{M})$ in the presence of cyclothiazide (CTZ;20 $\mu \mathrm{M})$, an antagonist of AMPAR desensitization, produced typical 
A

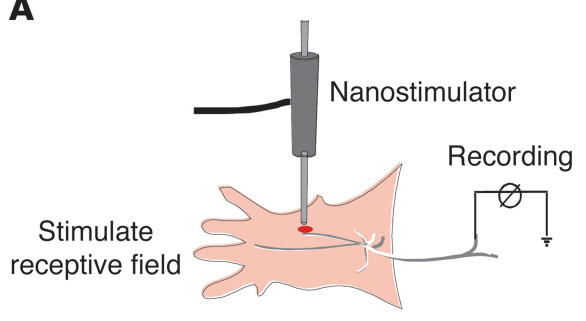

C

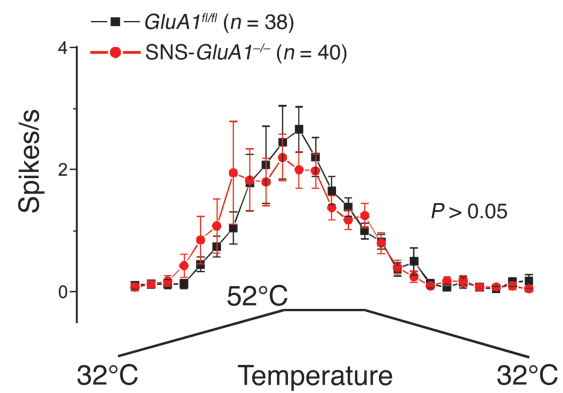

B
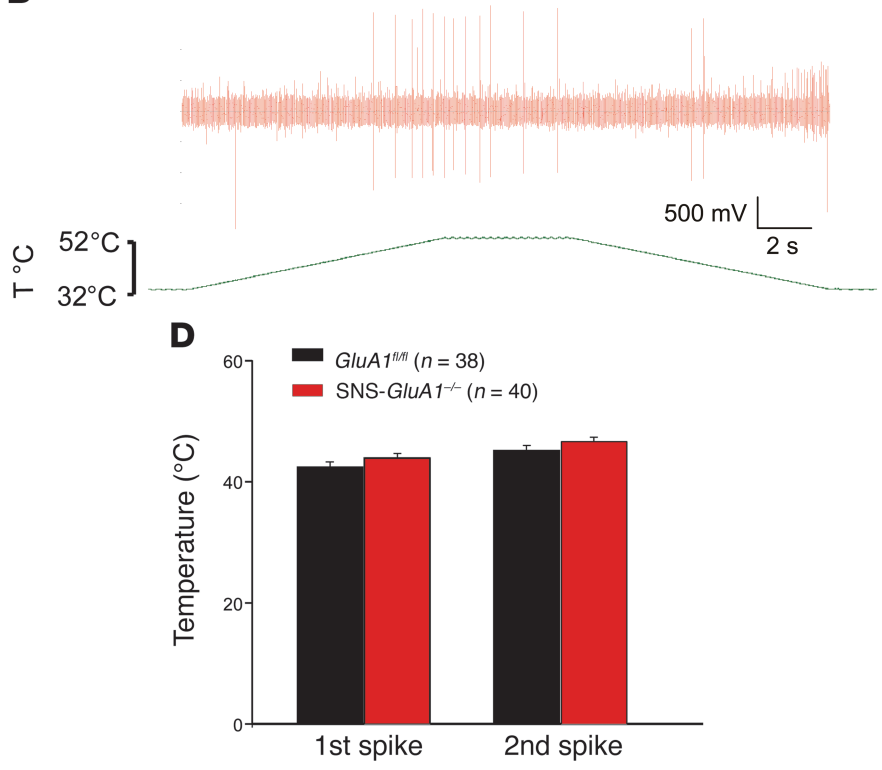

G
E
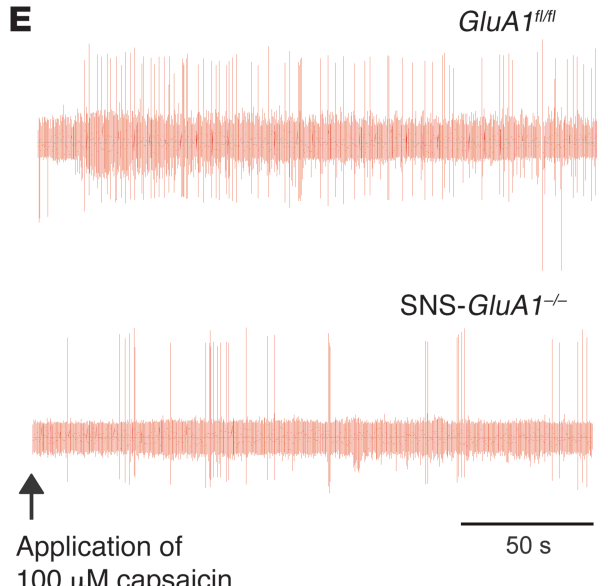

$100 \mu \mathrm{M}$ capsaicin
$\mathbf{F}$

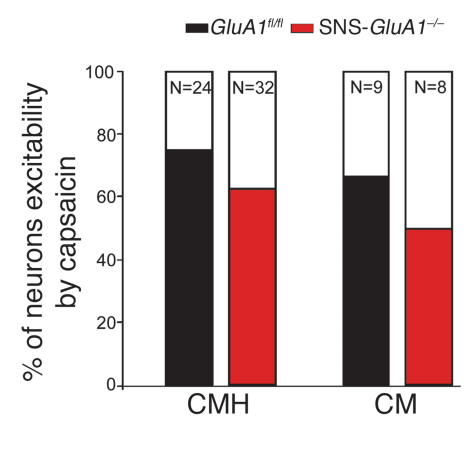

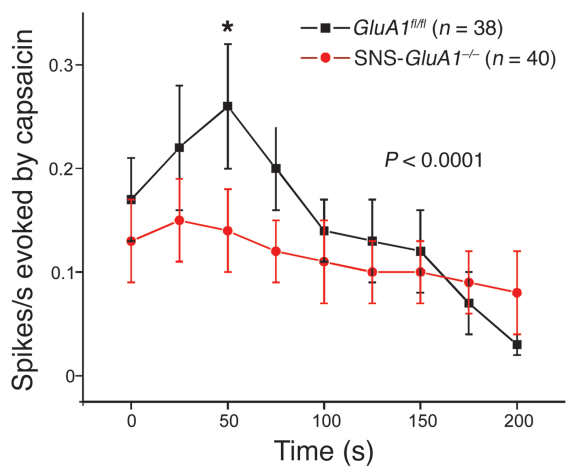

\section{Figure 3}

Electrophysiological analysis of peripheral nociceptive fiber responses to nociceptive stimuli in GluA1 $1^{t / f l}$ mice and SNS-GluA1-/- mice. (A) Scheme of the skin-saphenous nerve preparation and the recording setup (see Methods for details). (B and C) Analysis of responses of C-mechanoheat nociceptors to heat ramps. (B) A typical example of spike activity in response to a heat ramp. T, temperature. (C) Average spike activity from a population of nociceptors. (D) The threshold temperature for evoking first and second spikes. (E-G) Analysis of responses of C-mechanoheat $(\mathrm{C}-\mathrm{MH})$ and $\mathrm{C}$-mechano $(\mathrm{CM})$ types of nociceptors to application of capsaicin (100 $\mu \mathrm{M})$ to the receptive field in the skin. (E) Typical examples of

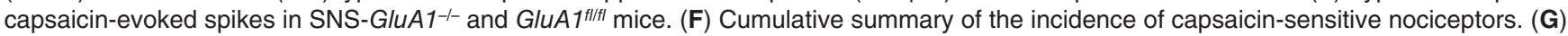
Summary of the magnitude of capsaicin-evoked action potentials in nociceptors of SNS-GluA1-/- and GluA $1^{f l / f l}$ mice. No significant differences were seen between genotypes in $\mathbf{C}, \mathbf{D}$, and $\mathbf{F}$. Two-way ANOVA for repeated measures revealed significant differences in the cumulative curves representing SNS-GluA1-/- and GluA1 ${ }^{f / f l}$ mice $(P<0.001) .{ }^{*} P<0.05$ for the time point indicated (post-hoc Bonferroni test).

increases in the ratio of Fura-2 fluorescence at $340 / 380 \mathrm{~nm}$ in 10 out of 44 nociceptive neurons from GluA1 fl/fl mice. In contrast, only 1 out of 46 neurons from SNS-GluA1-/- mice responded to AMPA plus CTZ (see Figure 4G for typical examples and Figure $4 \mathrm{H}$ for quantitative summary), although all neurons responded to $\mathrm{KCl}$ (Figure $4 \mathrm{H})$. The proportion of nociceptive neurons responding to glutamate $(3 \mathrm{mM})$ was significantly higher in $G l u A 1^{f l / f l}$ mice than that in SNS-GluA1 $1^{-/-}$mice $(P=0.02$; Figure $4 \mathrm{H})$. Furthermore, the magnitude of calcium gradients evoked by glutamate was reduced by nearly $50 \%$ in SNS-GluA1-/- mice as compared with that in GluA1flfl mice (see Figure 4G for typical examples and Figure 4I for quantitative summary). In contrast, the magnitudes of calcium responses induced by $\mathrm{KCl}$ (Figure 4I) were comparable in neurons

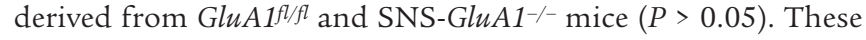
results show that a considerable proportion of nociceptive neurons express calcium-permeable AMPARs (at least 25\%) and that GluA1-containing calcium-permeable AMPARs account for about $50 \%$ of the calcium flux produced by the endogenous agonist glutamate in nociceptors.

Specific deficits in acute nociceptive bypersensitivity upon DRG-specific loss of GluA1 but not GluA2. Behavioral testing revealed that a loss of GluA1 in nociceptors was specifically associated with marked changes in pain-related behavior. In contrast, SNS-GluA2 $2^{-/-}$mice are indistinguishable from their control GluA2f/fl littermates in all 
A Resting membrane potential

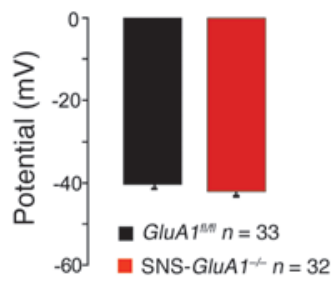

C

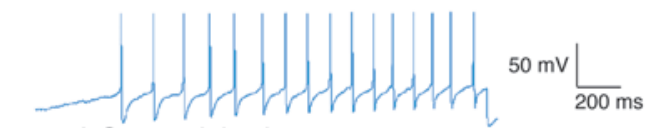

$400 \mathrm{pA}$ Current injection

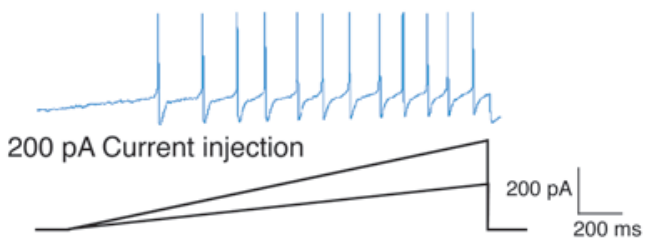

$\mathbf{F}$

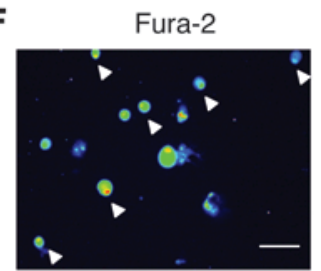

$\mathbf{B}$

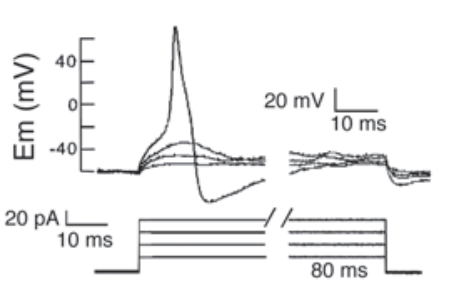

D
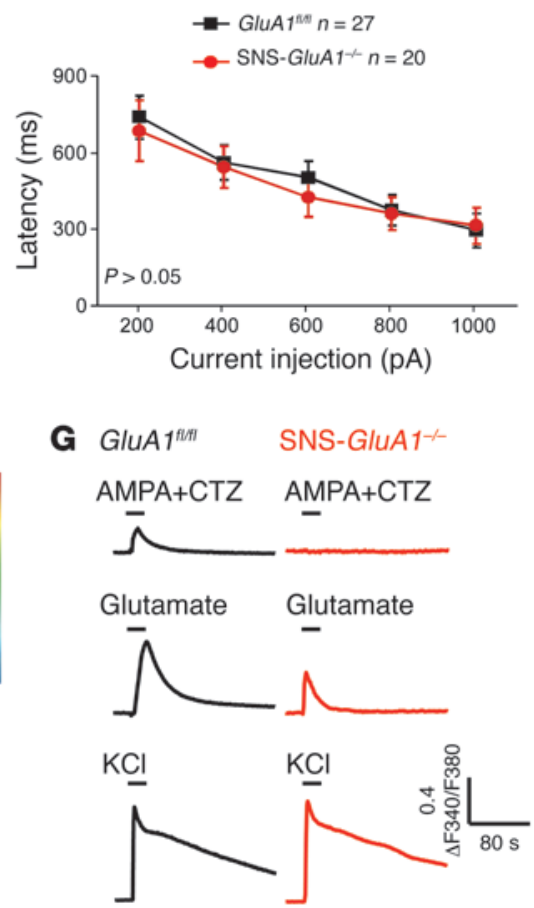

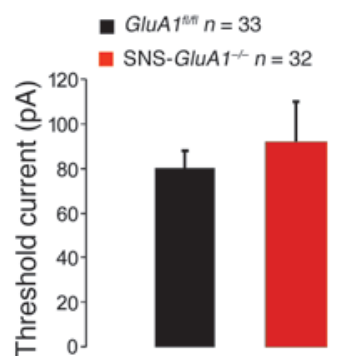

E
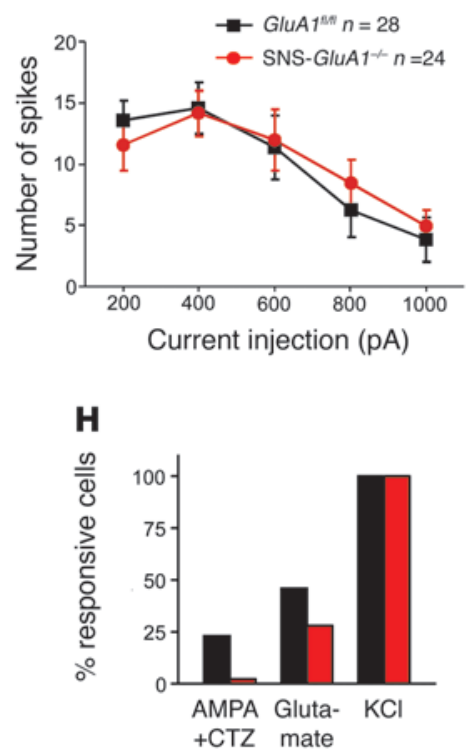

I

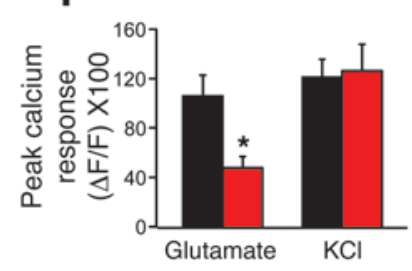

Figure 4

Analysis of the basal excitability, activation properties, and calcium transients in the somata of nociceptive sensory neurons in SNS-GluA1 ${ }^{-/-}$and GluA1 $1^{f \mid f l}$ mice. (A) Average resting membrane potential in nociceptive neurons identified via small cell size and humped action potential. (B) Typical example of action potentials (APs) generated by step-wise increments of current injection (threshold) and the average threshold current required to evoke action potentials. (C) Typical examples of the nature and frequency of action potentials evoked by prolonged current injections. (D and E) Input-output curves representing (D) the average latency to spiking and (E) the number of spikes in response to graded current injections. In A-E, $P>0.05$ between genotypes, 2-way ANOVA. (F) Typical examples of Fura-2-loaded dissociated DRG nociceptive neurons identified live via binding to $\mathrm{IB}_{4}$ (arrowheads). (G) Typical examples of traces of calcium transients represented as ratiometric change in fluorescence emission upon sequential excitation at $340 \mathrm{~nm}$ and $380 \mathrm{~nm}$ (F340/F380) prior to and after bath application of glutamate (3 mM), AMPA (10 $\mu \mathrm{M})$ plus CTZ $(20 \mu \mathrm{M})$, or $\mathrm{KCl}(25 \mathrm{mM}$, internal control). (H) Summary of the fraction of Fura-2-labeled IB 4 -positive DRG neurons responding to agonists (expressed as a percentage of total cells tested) over 9 to 10 independent culture experiments. (I) Average of peak ratiometric changes

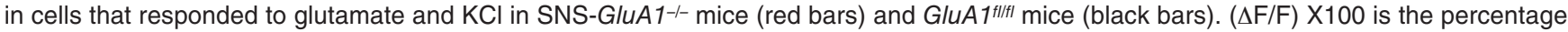
increase of F340/F380 ratio over basal. ${ }^{*} P<0.05$, ANOVA for random measures, Fisher's post-hoc test.

nociception tests used in this study (see Supplemental Figure 4). Acute withdrawal responses to pressure or to a heat ramp applied (using a plantar test or to constant heat at $50^{\circ} \mathrm{C}$ and $55^{\circ} \mathrm{C}$ ) were not significantly altered in SNS-GluA1-/- mice as compared with those in GluA1 fl/fl mice (Figure 5, A and B). However, acute nocifensive responses evoked by an intraplantar injection of capsaicin (Figure 5C) were markedly attenuated in SNS-GluA1-/- mice as compared with those in GluA1fl/fl mice, entirely consistent with our electrophysiological finding that capsaicin excites nocicep-

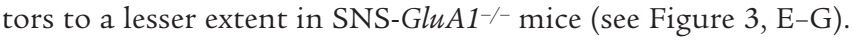
Application of capsaicin to the skin has been reported to lead to a rapid release of glutamate from C-fibers (e.g., refs. 23, 24). We observed that subcutaneous pretreatment of wild-type mice with GYKI 52466 (5 nmol), a specific AMPAR antagonist, significantly 
A
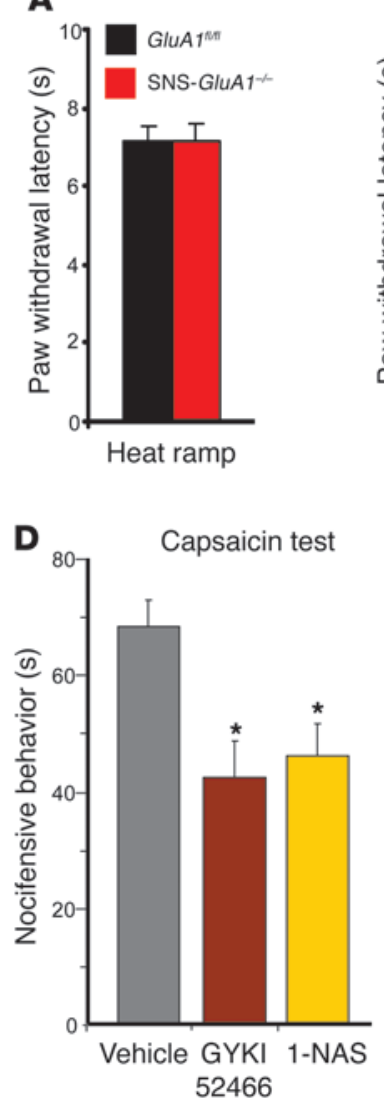

B

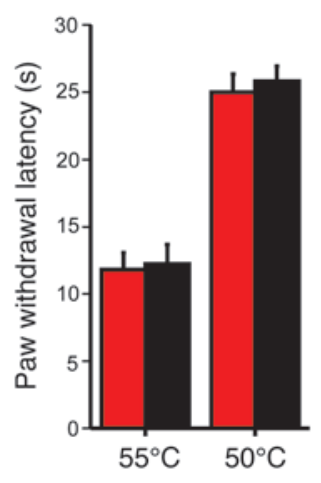

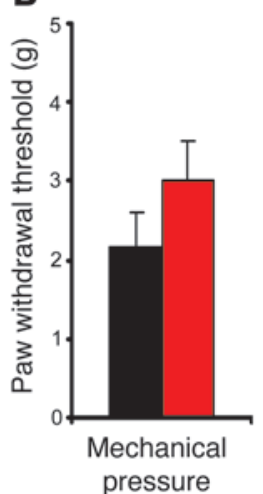

C

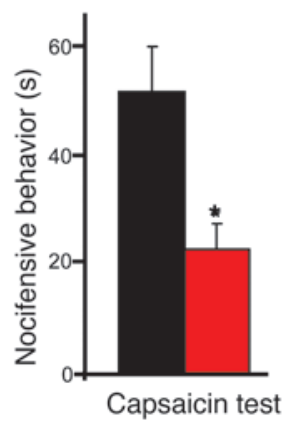

\begin{abstract}
E Formalin test
\end{abstract}

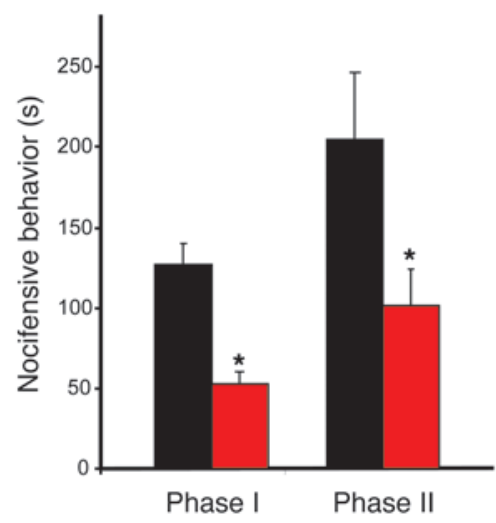

$\mathbf{F}$
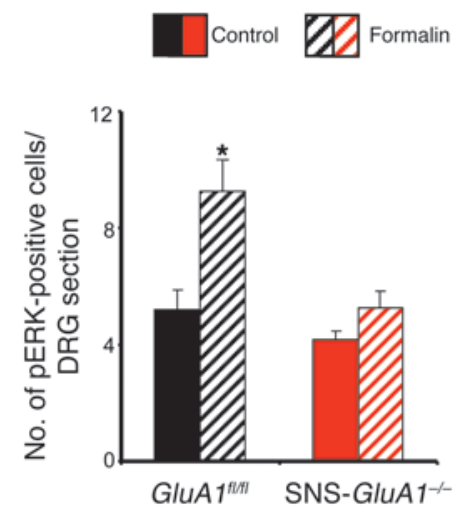

Figure 5

Analysis of the contribution of peripheral AMPARs to the modulation of acute nociception and early nociceptive hypersensitivity. (A) Latency of paw withdrawal after application of noxious heat in form of infrared heat ramp (left) or constant temperature (right) and (B) threshold of paw withdrawal to mechanical stimuli applied via the dynamic aesthesiometer is normal in SNS-GluA1-l- mice. (C) SNS-GluA1/- mice show reduced duration of acute nocifensive behaviors in seconds, upon hind paw intraplantar injection of $0.06 \%$ capsaicin $\left(n=7-15\right.$ mice per group; $\left.{ }^{*} P<0.05\right)$. (D) Capsaicin-induced nocifensive behaviors in wild-type mice are reduced upon peripheral pretreatment with a pan-antagonist of AMPARs, GYKI 52466, or a specific antagonist of calcium-permeable AMPARs, 1-NAS ( $n=5$ mice per group; $\left.{ }^{*}<0.05\right)$. (E) SNS-GluA1-/- mice show reduced duration of acute nocifensive behaviors (phase I) as well as early inflammatory hypersensitivity (phase II), upon hind paw intraplantar injection of $1 \%$ formalin $\left(n=7-15\right.$ mice per group; ${ }^{*} P<0.05$ as compared with GluA $1^{f / f f l}$ mice). (F) Quantification of neuronal activation 1 hour after intraplantar formalin injection by means of counting pERK-positive neurons in DRG sections of SNS-GluA1-/- and control mice $(n=7-15$ mice per group; ${ }^{\star} P<0.05$ as compared with the corresponding control mice). Data was analyzed via ANOVA for random measures, post-hoc Fischer's test in $\mathbf{E}$ and $\mathbf{F}$, and Student's $t$ test in $\mathbf{A}-\mathbf{D}$.

attenuated capsaicin-evoked nocifensive responses $(P=0.005$; Figure 5D). Similar results were observed upon peripheral treatment with 1-naphthyl acetyl spermine (1-NAS, 100 pml), which is postulated to function as a selective inhibitor of calcium-permeable AMPARs $(25)(P=0.01$; Figure 5D). This suggests that capsaicin-induced glutamate release activates GluA1-containing AMPARs, leading to augmentation and prolongation of the capsaicin-evoked excitation of C-fibers. Similar to the effects of capsaicin, intraplantar formalin-evoked acute nocifensive responses (phase I) occurred to a markedly lesser extent in SNS-GluA1-/- mice than in GluA1flfl mice (Figure 5E). Interestingly, formalin-induced increases in the number of DRG neurons expressing the plasticity-related molecules, phosphorylated ERK1/2 (26), occurred to a

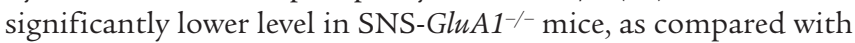
GluA1flfl mice (Figure 5F), suggesting that plasticity evoked by persistent nociceptive inputs in peripheral nociceptive neurons requires GluA1-containing AMPARs. Consistent with the above, the phase II behavioral response to formalin, which reflects early inflammatory hypersensitivity, was also significantly attenuated

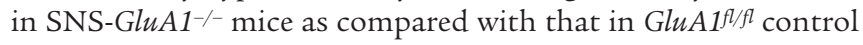
mice $(P=0.04$; Figure 5E).

Marked defects in the development and maintenance of chronic inflammatory mechanical hypersensitivity in SNS-GluA1-/- mice. We then addressed whether the manifestation of long-lasting inflammatory pain is functionally linked to AMPARs localized on nocicep-

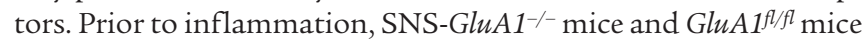
demonstrated comparable response thresholds (Figure 6A) and response frequencies (data not shown) to von Frey hairs applied to the plantar surface. After intraplantar injection of CFA unilaterally in the hind paw, SNS-GluA1 $1^{-/-}$mice developed paw edema, which was comparable to that shown by their respective control littermates (Supplemental Figure 5A). CFA-induced mechanical hypersensitivity was tested by applying graded punctuate pressure via von Frey hairs, and the minimum force that evoked withdraw- 
A
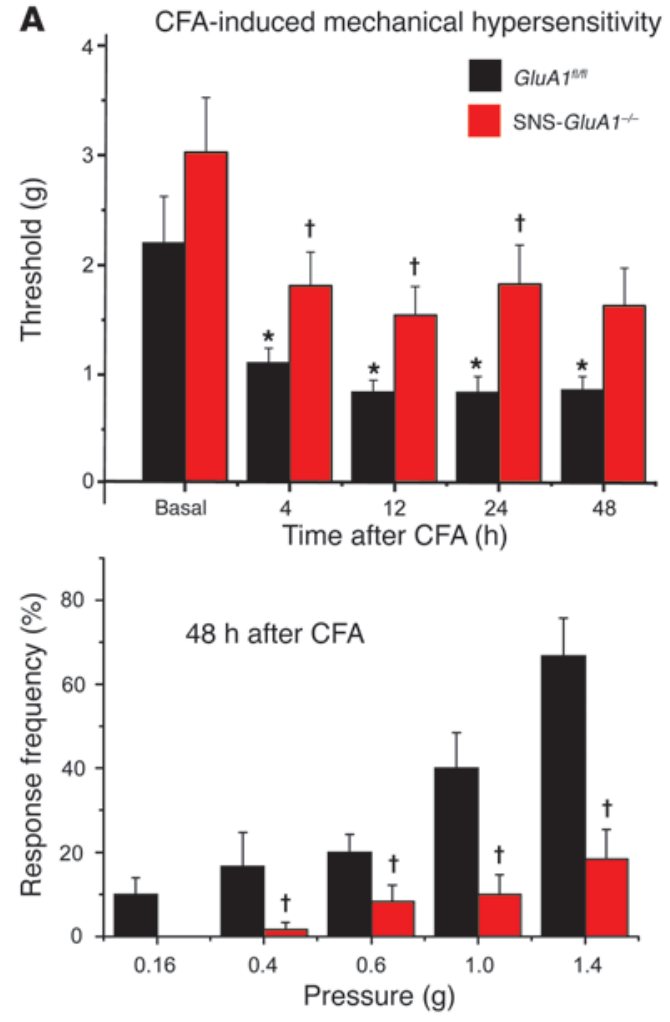

B CFA-induced thermal hyperalgesia

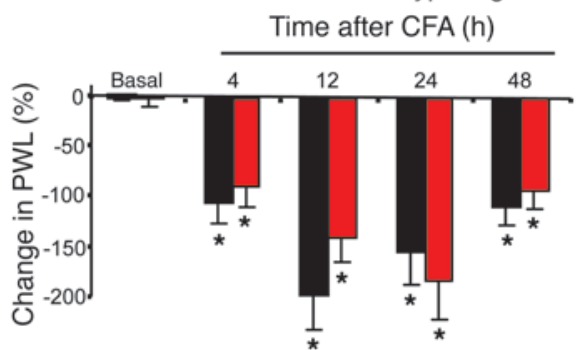

C
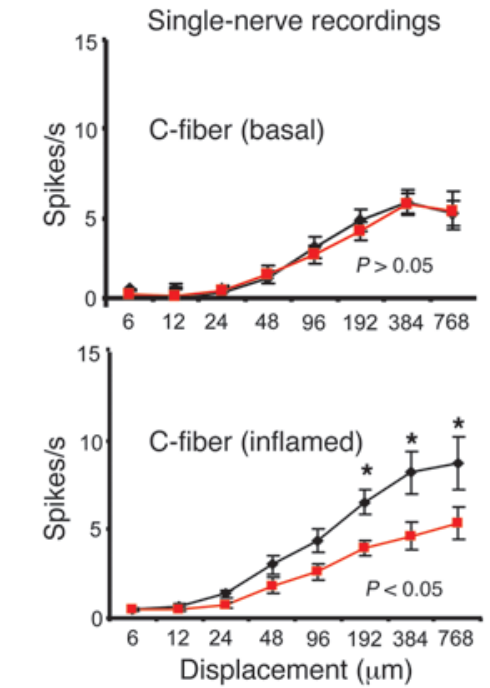
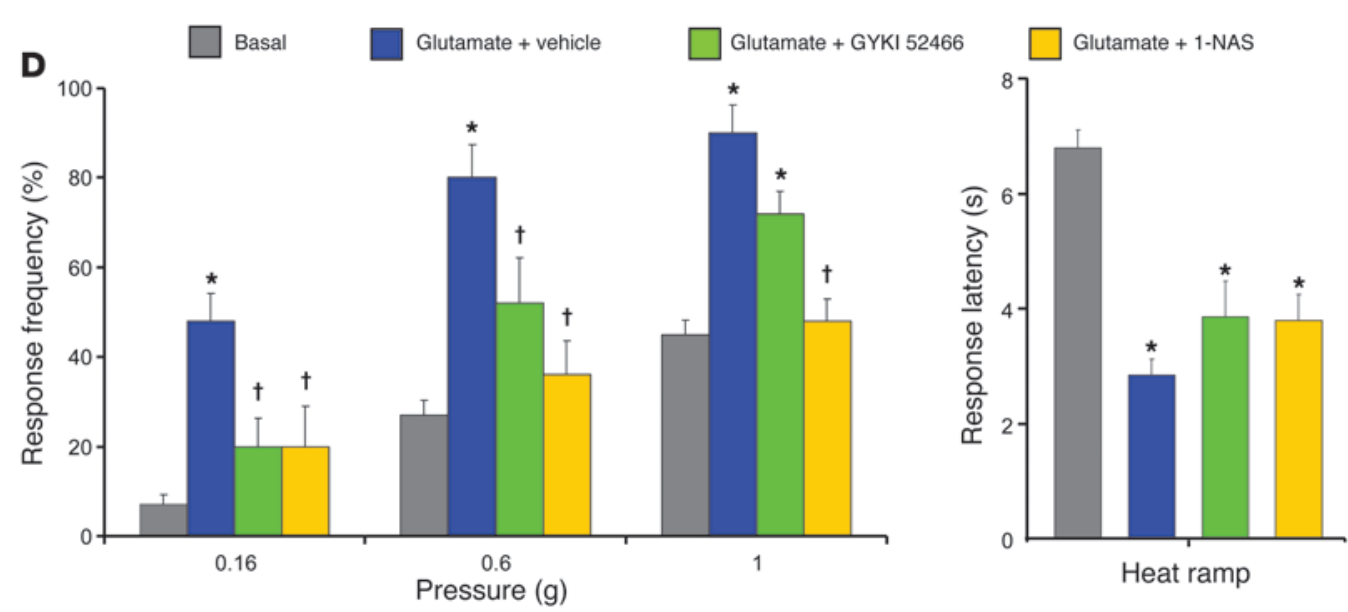

Figure 6

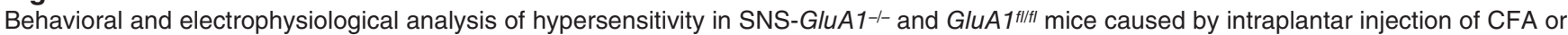
glutamate $(100 \mathrm{nmol})$. (A) Summary of response thresholds (defined as a force eliciting a response frequency of at least $40 \%)$, and a comparison of response frequencies in response to plantar von Frey hair application. (B) Changes in paw withdrawal latency (PWL) in response to infrared heat in the inflamed paw represented as the percentage decrease over the contralateral uninflamed paw. $(\mathbf{A}$ and $\mathbf{B}){ }^{\star} P<0.05$ as compared with the corresponding basal states; ${ }^{\dagger} P<0.05$ as compared with GluA $1^{f / f t}$ mice; ANOVA for random measures, post-hoc Fisher's test; $n=16$ per genotype. (C) Firing frequencies in response to pressure applied via a nanomotor (expressed in terms of displacement) in the skin-nerve preparation. After inflammation, C-mechanoceptors showed potentiated responses in GluA1 ${ }^{f l / f l}$ mice but not in SNS-GluA1-/- mice $\left({ }^{*} P<0.05\right.$, repeated measures ANOVA over the whole curve; ${ }^{*} P<0.05$ between the 2 genotypes at the indicated points, post-hoc Bonferroni; $n=10-20$ mice per group). (D) Peripherally administered GYKI 52466 as well as 1-NAS significantly blocked mechanical hypersensitivity, but not thermal hyperalgesia, evoked by intraplantar injection of glutamate. ${ }^{*} P<0.05$ as compared with basal; $\uparrow P<0.05$ as compared with glutamate plus vehicle group; $n=5$ mice per group. All data points represent mean \pm SEM. 
A
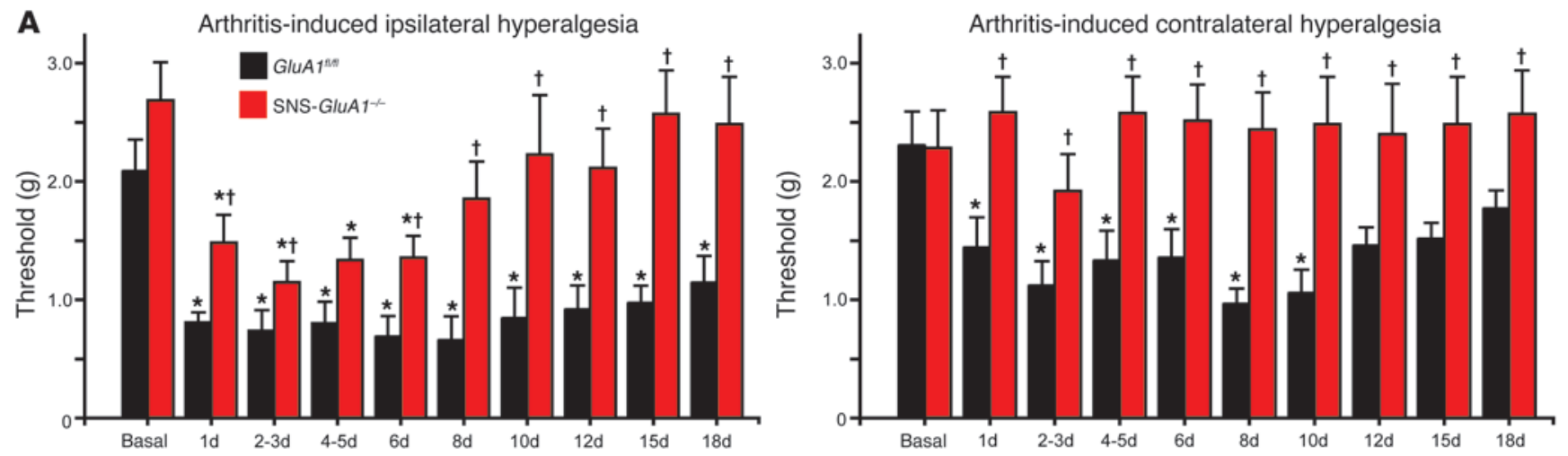

B

Arthritis-induced ipsilateral hyperalgesia
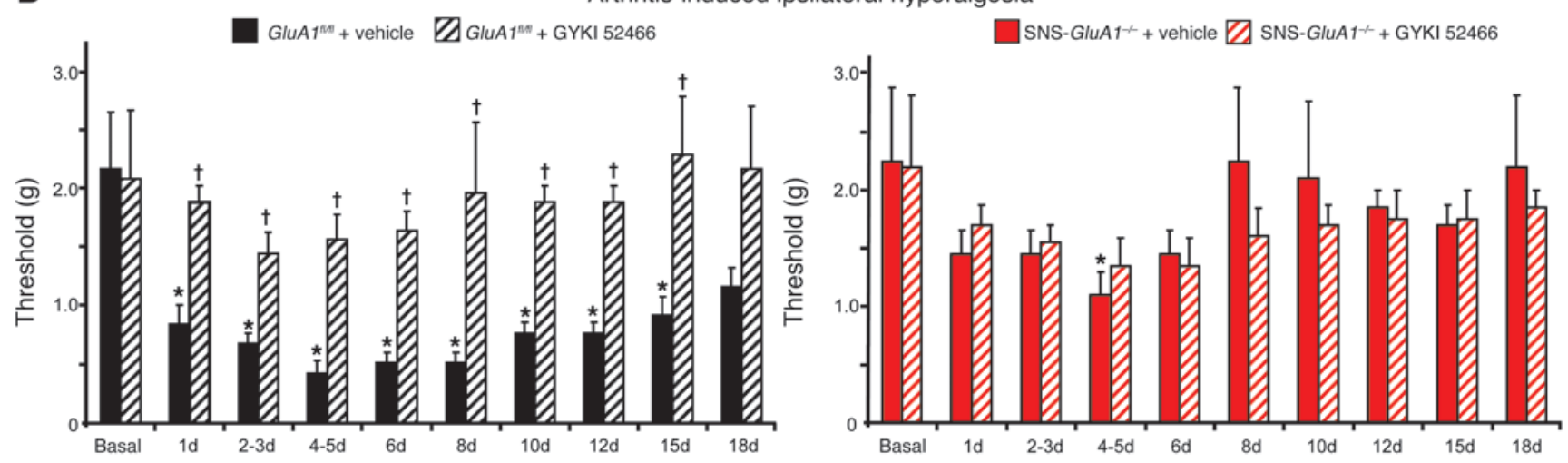

Figure 7

Analysis of arthritis-induced chronic pain hypersensitivity in GluA1 fl/fl mice and SNS-GluA1-l- mice. (A) Summary of response thresholds (defined as a force eliciting a response frequency of at least $40 \%$ ) to von Frey hair application prior to (basal) and up to 18 days after induction of kaolin-

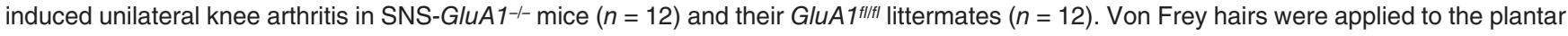
surface of the hind paws ipsilateral and contralateral to the arthritic knee. (B) Systemic treatment with the AMPAR antagonist, GYKI 52466, once per day completely blocked the development and maintenance of mechanical hypersensitivity (ipsilateral values are shown) in GluA1 $1^{f l f l}$ mice but not in SNS-GluA1-l- mice ( $n=6$ per group). (A and B) ${ }^{*} P<0.05$ as compared with basal; ${ }^{\dagger} P<0.05$ as compared with GluA1/lftl mice.

al responses toward at least 2 out of 5 applications of von Frey hairs was denoted as response threshold. Whereas GluA1flfl mice developed significant mechanical hypersensitivity (drop in von Frey hair threshold) at 4, 12, 24, and 48 hours after CFA injection as compared with their corresponding basal values (Figure 6A; ANOVA followed by Fisher's test), the drop in the mechanical response threshold in SNS-GluA1-1- mice over basal values was not statistically significant $(P>0.05$; Figure 6A). Furthermore, although von Frey thresholds in GluA1 fl/fl mice and SNS-GluA1-1mice under basal conditions were not significantly different from each other ( $P=0.3$, ANOVA followed by Fisher's test), response thresholds at 4, 12, 24, and 48 hours were significantly different from each other (Figure 6A; ANOVA followed by post-hoc Fisher's test). This was also evident when directly comparing the response frequencies to graded von Frey hairs between $G l u A 1^{f l / f l}$ mice and SNS-GluA1 $1^{-1-}$ mice; an example at 48 hours after CFA injection, a time of peak hyperalgesia, is shown in Figure 6A $(P<0.05$ between the 2 genotypes). In contrast to mechanical hyperalgesia, CFA-induced thermal hyperalgesia, which was calculated as percentage decrease in paw withdrawal latency in the inflamed paw over the uninflamed contralateral paw, developed in both SNSGluA1 $1^{-/}$mice and GluA1 $1^{f l f l}$ mice $(P<0.05$ as compared with basal; Figure 6B) to a similar level ( $P>0.05$ between genotypes). SNS-
GluA2 $2^{-/-}$mice did not differ from their GluA2flfl littermates with respect to CFA-induced thermal and mechanical hypersensitivity (Supplemental Figure 4).

Nociceptors lacking GluA1-containing AMPARs do not show mechanical sensitization upon peripheral inflammation. We directly studied the sensitivity of nociceptors to mechanical stimuli and inflammationinduced changes thereof in SNS-GluA1-/- and GluA1 fl/fl mice. Electrophysiological recordings were made from A- $\delta$ and C-fibers in the saphenous nerve, and the mechanical stimuli consisted of a series of ascending displacement stimuli applied to the receptive field of single fibers. A- $\delta$ nociceptors (Supplemental Figure 6) and C-fibers (Figure 6C) showed comparable threshold and suprathreshold

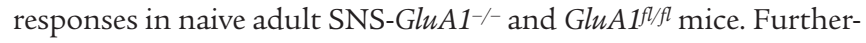
more, conduction velocities were not different between 2 genotypes (Student's $t$-test, $P>0.05$; Supplemental Table 2). C-mechanoceptors from GluA1 $1^{f l f l}$ mice demonstrated potentiated responses to suprathreshold displacement stimuli when tested at 4-6 hours after paw inflammation induced by injection of CFA $(P<0.05$, repeated measures ANOVA; Figure 6C). In contrast, C-mechanoceptors in SNS-GluA1 $1^{-/-}$mice did not show any significant sensitization to displacement stimuli after paw inflammation $(P=0.0117$, repeated ANOVA on the 2 curves; Figure 6C). The 2 genotypes were significantly different from each other at displacement values of $192 \mu \mathrm{m}$ 
$(P<0.05), 384 \mu \mathrm{m}(P<0.01)$, and $768 \mu \mathrm{m}(P<0.01$, Bonferroni post-test). Although A- $\delta$ fibers showed a tendency for sensitization to mechanical stimulation in CFA-injected GluA1 $1^{f l f l}$ mice, a repeated measures ANOVA analysis of the stimulus-response curves failed to reach statistical significance (Supplemental Figure 6).

Peripheral glutamate-evoked hyperalgesia. Intrigued by the observation that GluA1-containing AMPARs appeared to play a significant role in inflammation-induced mechanical hyperalgesia, but not thermal hyperalgesia, we asked whether this generally applies to all AMPARs. We observed that acute mechanical hyperalgesia evoked by intraplantar injection of glutamate (100 nmol; $20 \mathrm{~min}$ utes after injection) tested via von Frey hair application to the paw was significantly attenuated by peripheral pretreatment with a selective AMPAR antagonist, GYKI $52466(P=0.03$ for $0.16 \mathrm{~g}$ and $0.6 \mathrm{~g}$ force; Figure $6 \mathrm{D})$. In contrast, glutamate-induced acute thermal hyperalgesia was not significantly affected by peripheral GYKI 52466 (Figure 6D). Furthermore, glutamate-induced mechanical hypersensitivity in wild-type mice was blocked by peripherally administered 1-NAS, an inhibitor of calcium-permeable AMPARs, significantly at a dose of $100 \mathrm{pmol}$ and completely at a dose of $1 \mathrm{nmol}$ (Figure 6D), whereas glutamate-induced thermal hyperalgesia remained unaffected (Figure 6D, right). Importantly, 1-NAS-induced blockade of glutamate-induced peripheral sensitization was also observed in GluA fl/fl $^{l c o n t r o l}$ ) mice but not in SNS-GluA1 $1^{-/-}$mice, indicating thereby that at these doses peripheral 1-NAS produced its effects by specifically blocking peripheral calcium-permeable AMPARs (Supplemental Figure 7).

Arthritis-induced centrally mediated mechanical hypersensitivity does not develop in SNS-GluA1-/- mice. We went on to analyze nociceptive behavior in another clinically relevant model of chronic pain, namely unilateral knee-arthritis. This model is particularly useful for studying central changes elicited by enhanced excitatory drive from the periphery into the spinal cord, because the primary hyperalgesia evoked in the injured knee is accompanied by secondary hyperalgesia in the ipsilateral and contralateral hind paws (ref. 27 and Figure 7A, left). As compared with control GluA $1^{f / f l}$ mice, SNS-GluA1 $1^{-/}$mice also showed a significantly reduced drop in response thresholds to von Frey at the ipsilateral paw after knee injury $(P<0.05$ at all time points except $4-5$ days after induction of arthritis; Figure 7A) and a markedly reduced duration of chronic hypersensitivity (Figure 7A). Furthermore, whereas GluA1 fl/fl mice developed marked, long-lasting mechanical hypersensitivity in the contralateral paw after knee injury (Figure 7A, right), SNSGluA1 $1^{-1-}$ mice failed entirely to develop contralateral hyperalgesia. Importantly, intraperitoneal administration of GYKI 52466 in this arthritis model completely blocked the development of mechanical hypersensitivity in GluA1 $1^{f l f l}$ (control) mice but did not affect mechanical sensitivity in arthritic SNS-GluA1 $1^{-/-}$mice (Figure 7B), showing thereby that protective effects of GYKI 52466 are indeed mediated by GluA1-containing AMPARs in nociceptors. The above differences did not arise from differences in the degree of inflammation across genotypes, because typical indicators of arthritis-induced knee inflammation, such as an increase in knee circumference (Supplemental Figure 5C), increased synovial levels of myeloperoxidase (Supplemental Figure 5D), and neutrophil invasion in the synovial membrane (immunoreactivity for GR1, arrowheads in Supplemental Figure 5E), occurred at comparable levels in GluA1fl/fl mice and SNS-GluA1-/- mice. Thus, injury-evoked central changes in the processing of nociceptive inputs were markedly attenuated upon a specific loss of GluA1 in peripheral nociceptive neurons, suggesting that GluA1-containing AMPARs contribute significantly to the excitatory drive from the periphery into the central nervous system.

In support of this hypothesis, we observed that glutamate release in the spinal dorsal horn after peripheral stimulation of nociceptors via formalin was affected in SNS-GluA1-/- mice. Microdialysis catheters were chronically implanted through the spinal dorsal horns of SNS-GluA1 $1^{-/-}$mice and GluA1fl/fl mice in vivo (schematically represented in Supplemental Figure 8A), and both genotypes showed a significant increase of glutamate concentrations immediately after formalin injection (before versus maximum after formalin, $P<0.001$ and $P<0.0027$ for SNS-GluA1 $1^{-1-}$ and GluA1fl/fl mice) (Supplemental Figure 8B). Analysis of area under the concentrations versus time curves, which represents not only the maximum change but also the duration of increase in glutamate concentration after stimulation, showed that $G l u A 1^{f l / f l}$ mice, but not SNS-GluA1-/- mice, exhibited a significant increase over baseline levels over the first 25 minutes after formalin injection; basal values of glutamate release were not significantly different between the 2 genotypes (Supplemental Figure 8C).

Peripheral GluA1-containing AMPARs as pharmacological targets to attenuate inflammatory pain? Although a few studies have reported antinociceptive effects with nonspecific antagonists of non-NMDA glutamate receptors (e.g., NBQX and CNQX; refs. 28-30), very little is known about the specific role of peripheral AMPARs. We therefore performed experiments in which GYKI 52466 was administered to mice, either systemically (subcutaneous, $20 \mu \mathrm{mol} / \mathrm{kg}$ bodyweight) or peripherally (intraplantar hind pawinjection, $5 \mathrm{nmol}$ ), immediately after and at 18 and 23 hours after unilateral CFA injection in the paw. In wild-type mice, peripheral administration of GYKI 52466 significantly attenuated the development of mechanical hyperalgesia when tested 24 hours after CFA injection and measured using the von Frey hair test $(P<0.05$ as compared with basal; $P<0.05$ as compared with CFA plus vehicle; von Frey thresholds are shown in Figure 8A). In contrast, peripheral GYKI 52466 did not block the development of CFA-induced thermal hyperalgesia $(P<0.05$ as compared with basal; Figure $8 A)$. Similar to wild-type mice, GluA1 fl/fl mice treated peripherally with GYKI 52466 also showed markedly lower levels of mechanical hyperalgesia than vehicle-treated GluA1 ${ }^{f l f l}$ mice. In contrast, SNS-GluA1-/mice developed markedly lower levels of mechanical hyperalgesia than $G l u A 1^{f l f l}$ mice, as judged by measuring response frequency to von Frey hair stimulation, and pretreatment with peripheral GYKI 52466 did not further reduce behavioral signs of mechanical hyperalgesia compared with those of untreated SNS-GluA1-/- mice $(P<0.05$ as compared with basal; $P<0.05$ as compared with the GYKI 52466-treated group of the same genotype; $P<0.05$ between the genotype treated either with vehicle or GYKI 52466; Figure 8B). The same data is shown in Figure $8 \mathrm{C}$ in the form of an integration of responses with graded von Frey force (area under the curve). Similar results were obtained using a dynamic aesthesiometer to test mechanical sensitivity, and 2 routes of administration of GYKI 52466 were compared: GluA1fl/fl mice treated systemically or peripherally with GYKI 52466 developed less mechanical hyperalgesia than vehicle-treated mice 24 hours after CFA injection; SNS-GluA1-1- mice demonstrated less mechanical hyperalgesia after CFA injection than GluA1 $1^{f l f l}$ mice, and the antihyperalgesic effects of GYKI 52466 with both routes of administration were entirely lost in SNS-GluA1 ${ }^{-1-}$ mice $(P<0.05$ as compared with the vehicle-treated GluA1flfl group; $P<0.05$ as compared with the 

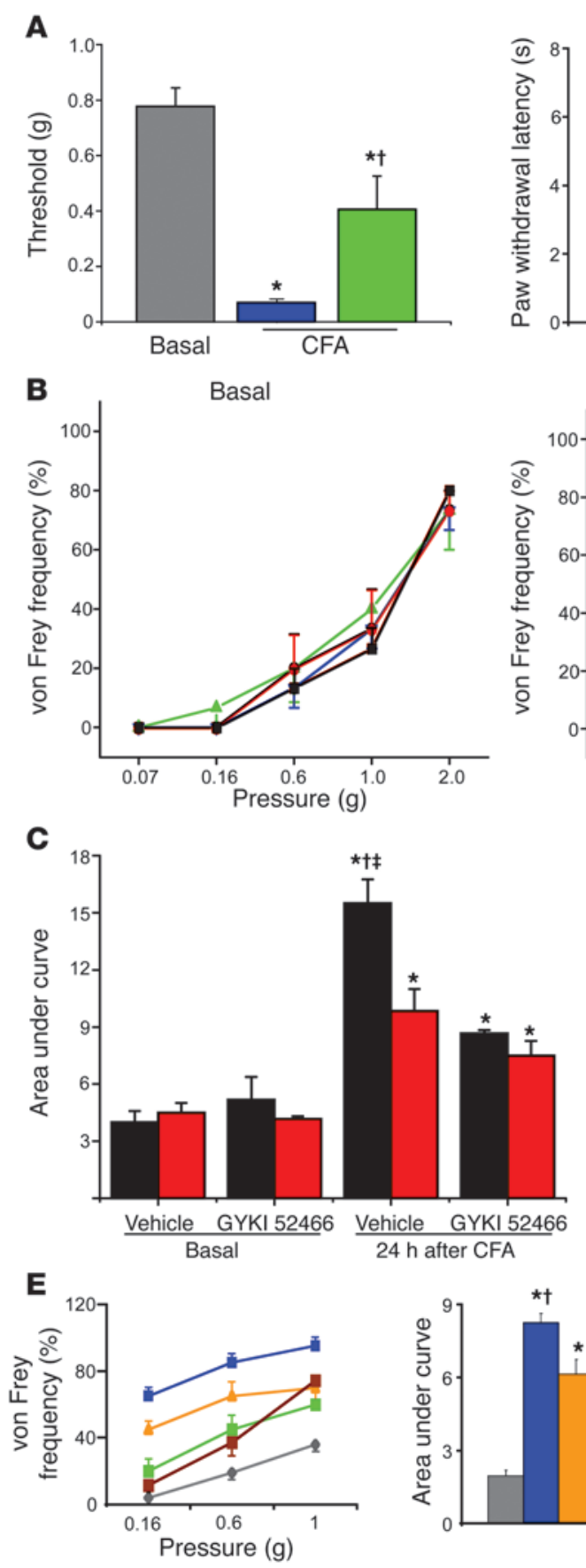

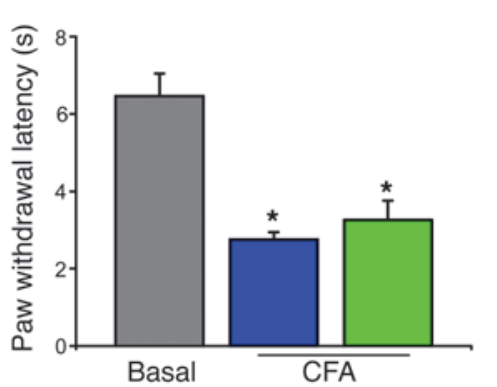

$24 \mathrm{~h}$ after CFA

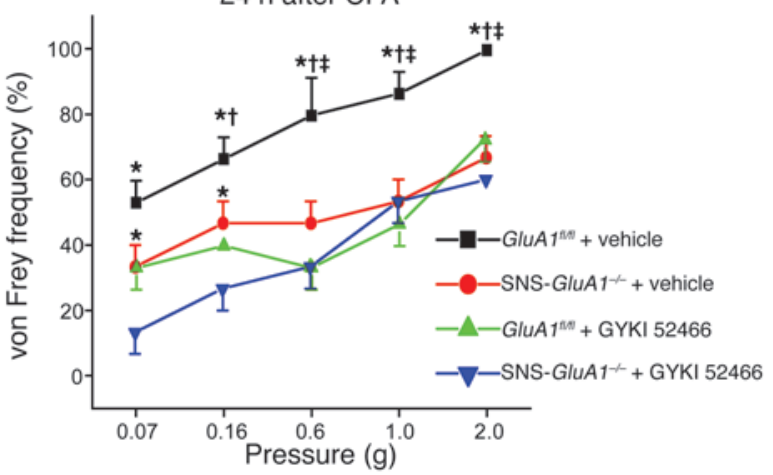

D GluA1 $1^{\text {III }}$

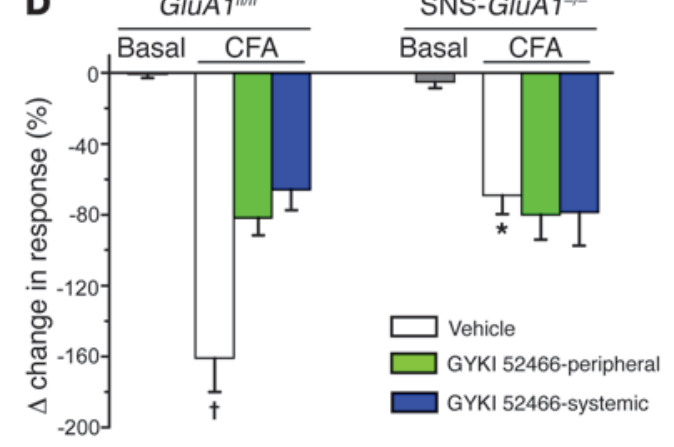

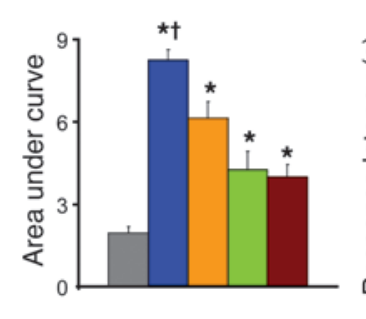

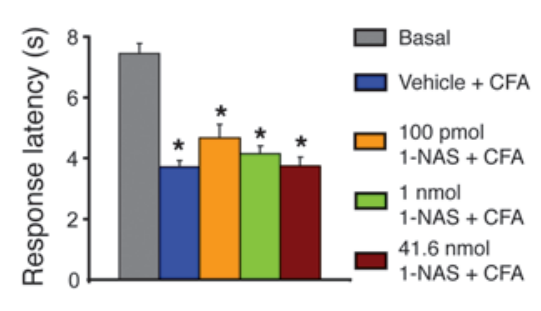

Figure 8

Effects of peripheral application of a selective AMPAR antagonist (GYKI 52466, $5 \mathrm{nmol}$ ) and a selective antagonist of calcium-permeable AMPARs (1-NAS, $100 \mathrm{pmol}, 1 \mathrm{nmol}$, and $41.6 \mathrm{nmol}$ ) on inflammatory hypersensitivity. (A) In wild-type mice, the CFA-induced drop in mechanical response threshold (plantar von Frey) is attenuated by peripheral GYKI 52466 (left), whereas CFA-induced thermal hyperalgesia is not affected (right). ${ }^{*} P<0.05$ as compared with basal values. ${ }^{\dagger} P<0.05$ as compared to vehicle group. (B and $\mathbf{C}$ ) Curves representing (B) response frequencies to graded von Frey hair stimulation and (C) corresponding integral values in the presence of GYKI 52466 or vehicle. (D) Analysis of mechanical hypersensitivity in the same cohorts of animals as in B and $\mathbf{C}$ using a dynamic aesthesiometer. CFA-induced mechanical hypersensitivity in the inflamed paw is represented as percentage decrease over the uninflamed paw. ${ }^{\dagger} P<0.05$ as compared with GYKI 52466 group; ${ }^{*} P<0.05$ as compared to vehicle treated GluA1 $1^{\text {flft }}$ group. (B and $\mathbf{C}$ ) ${ }^{*} P<0.05$ as compared with corresponding basal value; ${ }^{\dagger} P<0.05$ as compared with GYKI 52466 group; $¥ P<0.05$ as compared with SNS-GluA1-/- mice in the same group (GYKI 52466/vehicle); ANOVA followed by Fisher's post-hoc test, $n=6-10$ mice per group. (E) Effects of peripheral injection of 1-NAS on basal nociceptive sensitivity and CFA-induced hypersensitivity, expressed as shift in stimulus-response frequencies to plantar von Frey stimulation (left), the corresponding area under the curve (middle), and thermal hyperalgesia (right). 1-NAS dose-dependently attenuated CFA-induced mechanical hypersensitivity but not thermal hyperalgesia. ${ }^{\star} P<0.05$ as compared with corresponding basal value; ${ }^{\dagger} P<0.05$ as compared with the 1-NAS group; ANOVA followed by Fisher's post-hoc test, $n=5-10$ mice per group. 
vehicle-treated SNS-GluA1 ${ }^{-/}$group; Figure 8D). At doses that significantly affect these pain-related behaviors, GYKI 52466 did not cause sedation or affect motor functions, as measured using the open-field test (Supplemental Figure 9).

Similarly, CFA-induced mechanical hyperalgesia, but not thermal hyperalgesia, was dose-dependently attenuated when 1-NAS was administered peripherally to wild-type mice (as described above for GYKI 52466) $(P<0.05$ as compared with basal; $P<0.05$ as compared with the 1-NAS-treated group; Figure 8E). Taken together, these results show that peripheral application of an AMPAR antagonist or a specific blocker of calcium-permeable AMPARs inhibits inflammatory mechanical hypersensitivity by blocking GluA1-containing peripheral AMPARs, suggesting therapeutic relevance.

\section{Discussion}

It is well established that glutamate receptors in the central nervous system serve key functions in regulating plasticity events that underlie chronic pain states (1). This study serves toward clarifying the functional role of AMPARs in the peripheral arm of the pain pathway in physiological and inflammatory states and provides proof of principle for a therapeutic relevance of peripheral AMPARs. Importantly, we observed that selective deletion of individual AMPAR subunits in nociceptors, which alters their composition and properties without ablating AMPARs function completely, elicited specific changes in the processing of particular nociceptive modalities and algogens at peripheral terminals of nociceptors, while contributing to depression of neurotransmitter release at the central terminals of nociceptors. The net in vivo result of this differential nature of modulation at central and peripheral terminals of nociceptors clearly emerged as a pronociceptive, sensitizing role for GluA1-containing AMPARs in inflammatory hyperalgesia.

Hypersensitivity to mechanical stimuli represents one of the most debilitating and clinically relevant manifestations of pathological pain (1), which is why the finding that the composition and properties of peripheral AMPARs critically regulate mechanisms of mechanical hypersensitivity is very intriguing. Electrophysiological recordings in single nerve fibers showed that C-nociceptors in SNSGluA1 $1^{-/-}$mice fail to sensitize toward mechanical stimuli in inflamed tissues, although they responded normally under basal physiological conditions. These findings were corroborated by behavioral analyses in SNS-GluA1 $1^{-/-}$mice and with pharmacological tools, which consistently showed that blockade or loss of function of GluA1-containing AMPARs in peripheral nociceptors inhibits inflammatory mechanical hyperalgesia. Interestingly, this pertained not only to primary hyperalgesia but also to secondary mechanical hyperalgesia, a centrally maintained process (1), which was reduced in magnitude as well as in duration in mice lacking peripheral GluA1. This suggests that the GluA1-mediated increase in activity of peripheral nociceptors is causally linked to a potentiation of excitatory drive into the spinal cord, which would thereby facilitate central plasticity processes involved in secondary hyperalgesia.

Extrapolating from the excitatory nature of AMPARs in basal synaptic transmission at central synapses (2), one mechanistic explanation could be that peripheral GluA1-containing AMPARs contribute to the overall excitability of nociceptors. This is conceivable because a deletion of GluA1 can reduce AMPAR-mediated currents to some extent (3). However, several observations argue against this hypothesis. First, no differences were seen between SNS-GluA1-/mice and control mice, with respect to the resting membrane potential and threshold currents for evoking action potentials in patch-clamp recordings on the somata or basal response properties of peripheral nerves in the skin-nerve preparation. Furthermore, a change in general excitability of nociceptors would be expected to affect basal nociception in vivo and reduce hyperalgesia to all modalities of nociception, neither of which was reflected in behavioral phenotype of SNS-GluA1//- mice. We conclude therefore that GluA1-containing AMPARs do not generally regulate the excitability of nociceptors. Rather, our observations suggest that GluA1containing AMPARs specifically regulate the strength and duration of algogen-induced excitation of nociceptors. In this context, the finding that peripheral AMPARs modulate responsivity to capsaicin is particularly interesting, because it represents low frequency, persistent activation of nociceptors, such as that induced by inflammatory mediators, including TRPV1 agonists, such as lipids and protons $(31,32)$. In contrast to phasic, short-lasting thermal and mechanical nociceptive stimuli, capsaicin evokes responses that are persistent, long-lasting, and associated with repetitive spiking of nociceptors, and capsaicin application is known to evoke a rapid release of glutamate from activated C-fibers in the skin $(23,24)$. Taken together with our observation that inhibition of peripheral AMPARs significantly attenuated capsaicin-evoked nocifensive behaviors, this suggests that capsaicin-induced glutamate release augments the magnitude and duration of the capsaicin response by recruiting peripheral AMPARs. This can explain why responses to algogens, which repetitively activate and sensitize nociceptors, are modulated by changes in peripheral AMPARs, whereas basal nociceptive sensitivity to acute heat and mechanical is not.

The observations that a large population of AMPARs in nociceptors are permeable to calcium in wild-type mice and that reducing calcium permeability (e.g., in SNS-GluA1-/- mice or blockade with 1-NAS), but not increasing calcium permeability (e.g., in SNS-GluA2 $2^{-/}$mice), affects inflammatory hyperalgesia suggest that basal calcium flux via AMPARs in nociceptors may be the key determinant of inflammation-induced changes in nociceptor activation. Moreover, the observation that global GluA2 knockout mice, but not SNS-GluA2 $2^{--}$mice, showed exaggerated nociceptive hyperexcitability (6) suggests a larger contribution of centrally expressed GluA2-containing AMPARs toward modulation of sensitization processes. In inflamed tissues, algogen-induced release of glutamate and the ensuing activation of calcium-permeable AMPARs would lead to an enhanced calcium influx into nociceptors and thereby engage downstream calcium-dependent mechanisms that tend to increase action potential firing in the presence of algogens. In this context, a number of calcium-dependent protein kinases have been described, which serve to sensitize downstream receptors and channels, thereby enhancing excitability (32). This model can also explain why baseline excitability of nociceptors is not altered in SNS-GluA1-1- mice (see above), as such amplification mechanisms mediated by GluA1-containing AMPARs would only operate in conditions of inflammation when algogenic chemicals are present.

Unlike mechanical hyperalgesia, inflammatory thermal hyperalgesia was not affected in SNS-GluA1-/- mice. It has been proposed that TRPV1-expressing neurons, rather than the TRPV1 channel itself, are critical for heat sensitivity (31-34), and, per se, it cannot be ruled out that some TRPV1-expressing neurons are not targeted by the SNS-Cre mouse line used in this study. However, the observation that peripheral application of GYKI52466 or 1-NAS, which should similarly affect all sensory fibers innervating the injected zone, showed similar effects suggests that differences in types of 
sensory neurons affected by the genetic manipulation may not fully account for the above observations. Furthermore, previous studies have reported that much higher concentrations of peripherally applied glutamate are required to evoke thermal hyperalgesia than mechanical hyperalgesia (e.g., ref. 35). Furthermore, previous studies have suggested that metabotropic glutamate receptors are stronger modulators of mechanisms governing thermal hyperalgesia than ionotropic glutamate receptors $(36,37)$.

Although there is compelling ex vivo evidence for a role for presynaptic AMPARs on central terminals in inhibiting evoked transmission onto spinal neurons (9), the net change in excitation levels in the spinal dorsal horn and the in vivo relevance of this phenomenon are unclear. Here, we observed that although AMPA-induced inhibition of evoked transmission on spinal neurons was partially reduced in SNS-GluA1-/- mice, these mice did not show increased pain-related behavior, suggesting that presynaptic modulation via GluA1-containing AMPARs on spinal terminals of primary afferents may not play an important role in nociception in vivo. In the light of this dual modulation of nociceptor function by AMPARs, the level of glutamatergic tone may be a key determinant of how AMPARs affect nociceptor excitation. This is suggested by our observations that phenotypic differences between AMPAR mutant and control mice come about only in states associated with increased glutamatergic tone, e.g., following peripheral inflammation or repetitive activation of nociceptors via formalin or capsaicin. Mechanistically, this may not only result from the peripheral mechanisms discussed above but also from the phenomenon of dorsal root reflexes (DRRs), which are centrally triggered action potentials evoked by depolarization of spinal terminals of primary afferents (38). DRRs have been reported to occur in inflammatory pain states and contribute to neurogenic inflammation $(11,38)$. Besides GABA-A receptors, non-NMDA glutamate receptors have been shown to contribute to DRRs $(11,38)$. It is conceivable therefore that the availability of the endogenous agonist directly influences the relative contributions of central and peripheral GluA1containing AMPARs and determines whether antinociceptive or pronociceptive outcomes prevail.

In this context, it is interesting to note that in pathophysiological states, glutamate levels also rise markedly in peripheral tissues. For example, in addition to efferent release from activated primary afferents, invading macrophages, and serum from leaky blood vessels, Schwann cells as well as keratinocytes can release glutamate in inflamed tissue (11). Glutamate concentrations have been reported to rise dramatically in synovial fluid taken from arthritic patients (39), and the expression of AMPAR subunits in peripheral nerves is increased in inflamed tissues (11), thereby indicating agonist availability for activation of peripheral AMPARs in pathophysiological states. However, under these circumstances, it is to be expected that the concentration of glutamate in inflamed or injured tissue remains persistently elevated, in contrast to synaptically released glutamate, which is rapidly cleared away from the vicinity of synaptic glutamate receptors. Because native AMPARs exhibit rapid desensitization in the sustained presence of glutamate (40), this raises doubts about the extent to which AMPARs on peripheral nociceptors can stably influence nociceptor function upon prolonged exposure to glutamate. However, in cultured DRG neurons, AMPARs have been suggested to contribute more dominantly to glutamate-induced action potentials and steady-state depolarization and recover from desensitization more quickly than kainate receptors (41). Furthermore, diverse factors, such as RNA editing at the R/G site, alternative splicing of the flip and flop module, and heterogeneity in the composition of AMPAR/TARP complexes, are known to affect AMPAR desensitization recovery rates (42).

In summary, the results of this study demonstrate an important contribution of GluA1-containing calcium-permeable AMPARs expressed in the peripheral nervous system in modulating the activation properties of nociceptive neurons in response to algogens or in an inflammatory milieu. Furthermore, GluA1-containing AMPARs can control the inflow of nociceptive impulses from the periphery into the central nervous system and thus impact both peripheral and central hyperexcitability processes. These results provide a basis for developing novel pharmacological approaches targeting GluA1containing AMPARs in the periphery, which may enable alleviating inflammatory pain devoid of central side effects.

\section{Methods}

Details on methods are given in the Supplemental Methods.

Genetically modified mice. Homozygous mice carrying the floxed alleles (i.e., key loci flanked by loxP sites) for the mouse Gria1 locus encoding GluA1 $\left(G \ln A 1^{f / f l}\right.$ mice; ref. 15) and the mouse Gria2 locus encoding GluA2 (GluA2 $2^{f / f l}$ mice; ref. 16) were crossed with SNS-Cre mice (14), which express the Cre recombinase under the influence of the mouse Scn10a promoter (encoding Nav1.8) to obtain litters consisting of GluA1/l/f $\mathrm{SNS}-\mathrm{Cre}^{+}$mice (referred to

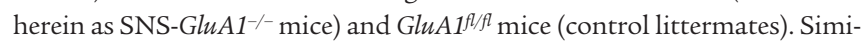
larly, GluA2 $2^{f / f l}$ mice were crossed with SNS-Cre mice to obtain litters consisting of $G l u A 2^{\ell / / f} \mathrm{SNS}^{-C r e^{+}}$mice (referred to herein as SNS-GluA2-/- mice) and $G l u A 2^{f / f l}$ mice (control littermates). Mice of all genotypes were individually backcrossed into the $\mathrm{C} 57 \mathrm{BL} / 6$ background for more than 8 generations before being crossed with each other. In all experiments, littermates were strictly used to control for genetic effects of the background.

Immunohistochemistry. Immunohistochemistry was performed using standard protocols and the following antibodies: rabbit polyclonal antiGluA2/3 and anti-GluA1 antisera (both from Chemicon International); rabbit polyclonal anti-CGRP antiserum (ImmunoStar); rabbit anti-phospho-p44/42 MAP Kinase (Thr202/Tyr204; pERK) antibody (Cell Signaling Technology); and an anti-Cre (69050, Novagen). Synaptic contacts of primary afferents in the spinal dorsal horn were analyzed on Vibratome sections as described before (7) using a rabbit PSD-95 antibody (a gift from M. Watanabe, Hokkaido University School of Medicine, Sapporo, Japan; ref. 43). Neutrophils were labeled in frozen sections of intraarticular synovial membrane of the mouse knee using a rat anti-Gr-1 (clone RB6-8C5, catalog 553125, BD Pharmingen; ref. 44).

Behavioral analysis. All animal use procedures were reviewed and approved by the local governing body (Regierungspräsidium Karlsruhe, Germany). All behavioral measurements were done in awake, unrestrained, and agematched adult (more than 3-month-old) mice of both sexes by individuals who were blinded to the genotype of the mice being analyzed. Mice were acclimatized to the experimental setups several times before the analysis. Nociceptive testing in mouse models of acute and chronic pain was performed as described in detail previously (refs. 6, 14, and Supplemental Methods).

Application of drugs. All chemicals were obtained from Sigma-Aldrich, except bicuculline, SYM 2081, and AMPA, which were obtained from Tocris Cookson Ltd. Mice were injected with GYKI 52466 and 1-NAS dissolved in DMSO and PBS, respectively. Subcutaneous injections of $20 \mu \mathrm{mol} / \mathrm{Kg}$ body weight of GYKI 52466 and intraplantar injections of GYKI $52466(5 \mathrm{nmol})$ and 1-NAS $(0.1,1$, and $41.6 \mathrm{nmol})$ were done in a volume of $20 \mu \mathrm{l}$ each, under light ether anaesthesia lasting for 30 seconds to 1 minute. In the CFA model, GYKI 52466 and 1-NAS were administered immediately after and at 18 hours and 23 hours after unilateral hind paw injection of CFA. No effects were observed with injections of vehicle only on nociceptive withdrawal responses. 
Skin-nerve preparation and single-fiber recordings. Single-nerve recordings were performed from nerve fibers identified on the basis of response properties, using the saphenous nerve-paw skin preparation as described in detail previously (refs. 21, 22, and Supplemental Methods).

Analysis of glutamate levels in the spinal cord. A microdialysis-based method was used to determine the change in glutamate levels in the spinal dorsal horn of adult mice after intraplantar injection of formalin as described previously (ref. 45 and Supplemental Methods).

Calcium imaging from cultured DRG neurons. DRG neurons were dissociated from (6- to 8-week-old) mice and loaded 24-48 hours later with Fura-2 $(10 \mu \mathrm{M})$ and Alexa 488-conjugated $\mathrm{IB}_{4}$-Fluor $(10 \mu \mathrm{g} / \mathrm{ml}$; Molecular Probes $)$ for live identification of small-diameter neurons. Calcium signals were recorded at a rate of $1 \mathrm{~Hz}$, and $\mathrm{F} 340 / 380$ ratios were calculated as described previously (ref. 46 and Supplemental Methods).

Patch-clamp recordings. Patch-clamp recording in whole-cell configuration was performed on visually identified lamina I and II neurons at room temperature in spinal slices prepared from mice at P15-P21 in response to dorsal root stimulation $(50-500 \mu \mathrm{A}, 0.1 \mathrm{~ms})$ as described in ref. 9 (Supplemental Methods). Patch-clamp recordings were done on dissociated DRG neurons 24 hours after preparation as described in Supplemental Methods. For determination of nociceptors, action potentials were evoked by repetitive $80 \mathrm{~ms}$ current injections, increasing from $20 \mathrm{pA}$ to $400 \mathrm{pA}$ in increments of $20 \mathrm{pA}$. The amount of current required for the first action potential was measured as threshold current. For excit- ability measurements, a 2-second current ramp, ranging from $200 \mathrm{pA}$ to $1,000 \mathrm{pA}$, was injected to the cell soma.

Statistics. All data are presented as mean \pm SEM. The 2-tailed Student's $t$-test or the ANOVA for random measures followed by post-hoc Fisher's least significant difference test were used to determine statistically significant differences $(P \leq 0.05)$.

\section{Acknowledgments}

The authors are grateful to Susan Carlton for discussions and insightful comments on an earlier version of the manuscript, Hans-Joseph Wrede and Dunja Baumgartner-Ahlert for technical assistance, and Rose LeFaucheur for secretarial help. This work was supported by grants from DFG to R. Kuner and G.R. Lewin, Italian MIUR to R. Bardoni, and Wellcome Trust to A.J. Todd, and NIH/NS029797 to A.B. MacDermott. R. Wang was supported by the DFG Research Training Group "Neuroinflammation."

Received for publication August 27, 2010, and accepted in revised form January 12, 2011.

Address correspondence to: Rohini Kuner, Pharmacology, Heidelberg University, Im Neuenheimer Feld 366, Room 204, Heidelberg, 69120, Germany. Phone: 49.6221.548289; Fax: 49.6221.548549; E-mail: rohini.kuner@pharma.uni-heidelberg.de.
1. Woolf CJ, Salter MW. Neuronal plasticity: Increasing the gain in pain. Science. 2000;288(5472):1765-1768.

2. Malinow R, Malenka RC. AMPA receptor trafficking and synaptic plasticity. Annu Rev Neurosci. 2002;25:103-126.

3. Seeburg PH, Single F, Kuner T, Higuchi M, Sprengel R. Genetic manipulation of key determinants of ion flow in glutamate receptor channels in the mouse. Brain Res. 2001;907(1-2):233-243.

4. Burnashev N, Monyer H, Seeburg PH, Sakmann B. Divalent ion permeability of AMPA receptor channels is dominated by the edited form of a single subunit. Neuron. 1992;8(1):189-198.

5. Engelman HS, Allen TB, MacDermott AB. The distribution of neurons expressing calcium-permeable AMPAR in the superficial laminae of the spinal cord dorsal horn. J Neurosci. 1999;19(6):2081-2089.

6. Hartmann B, et al. The AMPA receptor subunits, GluA1 and GluA2 reciprocally modulate spinal synaptic plasticity and inflammatory pain. Neuron. 2004:44(4):637-650.

7. Nagy GG, Al-Ayyan M, Andrew D, Fukaya M, Watanabe $M$, Todd AJ. Widespread expression of the AMPA receptor GluR2 subunit at glutamatergic synapses in the rat spinal cord and phosphorylation of GluR1 in response to noxious stimulation revealed with an antigen-unmasking method. J Neurosci. 2004;24(25):5766-5777.

8. Coggeshall RE, Carlton S. Ultrastructural analysis of NMDA, AMPA, and kainate receptors on unmyelinated and myelinated axons in the periphery. J Comp Neurol. 1998;391(1):78-86.

9. Lee CJ, et al. Functional expression of AMPAR on central terminals of rat dorsal root ganglion neurons and presynaptic inhibition of glutamate release. Neuron. 2002;35(1):135-146.

10. Lu CR, Hwang SJ, Phend KD, Rustioni A, Valtschanoff JG. Primary afferent terminals in spinal cord express presynaptic AMPAR. J Neurosci. 2002;22(21):9522-9529.

11. Carlton SM. Peripheral excitatory amino acids. Curr Opin Pharmacol. 2001;1(1):52-56.

12. Nagy A. Cre recombinase: the universal reagent for genome tailoring. Genesis. 2000;26(2):99-109.

13. Agarwal N, Offermanns S, Kuner R. Conditional gene targeting in neurons of the dorsal root ganglia and trigeminal ganglia. Genesis. 2004;38(3):122-129.

14. Agarwal N, et al. Nociceptor-specific conditional gene deletion reveals that cannabinoids mediate analgesia largely via peripheral type 1 cannabinoid receptors. Nature NeuroScience. 2007;10(7):870-879.

15. Zamanillo D, et al. Importance of AMPAR for hippocampal synaptic plasticity but not for spatial learning. Science. 1999;284(5421):1805-1811.

16. Shimshek DR, et al. Forebrain-specific glutamate receptor B deletion impairs spatial memory but not hippocampal field long-term potentiation. J Neurosci. 2006;26(33):8428-8440.

17. Fitzgerald M, Jennings E. The postnatal development of spinal sensory processing. Proc Natl Acad Sci U S A. 1999;96(14):7719-7722.

18. Lechner SG, Frenzel H, Wang R, Lewin GR. Developmental waves of mechanosensitivity acquisition in sensory neuron subtypes during embryonic development. EMBO J. 2009;28(10):1479-1491.

19. Bekkers JM, Stevens CF. Presynaptic mechanism for long-term potentiation in the hippocampus. Nature. 1990;346(6286):724-729.

20. Korn H, Faber DS. Quantal analysis and synaptic efficacy in the CNS. Trends Neurosci. 1991; 14(10):439-445.

21. Milenkovic N, Wetzel C, Moshourab R, Lewin GR. Speed and temperature dependences of mechanotransduction in afferent fibers recorded from the mouse saphenous nerve. J Neurophysiol. 2008;100(5):2771-2783.

22. Lechner SG, Lewin GR. Peripheral sensitisation of nociceptors via G-protein-dependent potentiation of mechanotransduction currents. J Physiol. 2009;587(pt 14):3493-3503.

23. Ueda M, Kuraishi Y, Sugimoto K, Satoh M. Evidence that glutamate is released from capsaicinsensitive primary afferent fibers in rats: study with on-line continuous monitoring of glutamate. Neurosci Res. 1994;20(3):231-237.

24. Medvedeva YV, Kim MS, Usachev YM. Mechanisms of prolonged presynaptic $\mathrm{Ca} 2+$ signaling and glutamate release induced by TRPV1 activation in rat sensory neurons. J Neurosci. 2008; 28(20):5295-5311.

25. Koike M, Iino M, Ozawa S. Blocking effect of 1naphthyl acetyl spermine onCa2+-permeable AMPA receptors in cultured rat hippocampal neurons, Neurosci Res. 1997;29(1):27-36.

26. Dai Y, et al. Phosphorylation of extracellular signalregulated kinase in primary afferent neurons by noxious stimuli and its involvement in peripheral sensitization. J Neurosci. 2002;22(17):7737-7745.

27. Bar KJ, et al. Changes in the effect of spinal prostaglandin E2 during inflammation: prostaglandin E (EP1-EP4) receptors in spinal nociceptive processing of input from the normal or inflamed knee joint. J Neurosci. 2004;24(3):642-651.

28. Nishiyama T, Gyermek L, Lee C, Kawasaki-Yatsugi S, Yamaguchi T. The systemically administered competitive AMPA receptor antagonist, YM872, has analgesic effects on thermal or formalin-induced pain in rats. Anesth Analg. 1999;89(6):1534-1537.

29. Stanfa LC, Dickenson AH. The role of non-N-methyl-D-aspartate ionotropic glutamate receptors in the spinal transmission of nociception in normal animals and animals with carrageenan inflammation. Neuroscience. 1999;93(4):1391-1398.

30. Chun YH, Frank D, Lee JS, Zhang Y, Auh QS, Ro JY. Peripheral AMPAR contribute to muscle nociception and c-fos activation. Neurosci Res. 2008; 62(2):97-104

31. Basbaum AI, Bautista DM, Scherrer G, Julius D. Cellular and molecular mechanisms of pain. Cell. 2009;139(2):267-284.

32. Huang J, Zhang X, McNaughton PA. Inflammatory pain: the cellular basis of heat hyperalgesia. Curr Neuropharmacol. 2006;4(3):197-206.

33. Cavanaugh DJ, et al. Distinct subsets of unmyelinated primary sensory fibers mediate behavioural responses to noxious thermal and mechanical stimuli. Proc Natl Acad Sci U S A. 2009;106(22):9075-9080.

34. Woodbury CJ, et al. Nociceptors lacking TRPV1 and TRPV2 have normal heat responses. J Neurosci. 2004;24(28):6410-6415.

35. Carlton SM, Zhou S, Coggeshall RE. Evidence for the interaction of glutamate and NK1 receptors in the periphery. Brain Res. 1998;790(1-2):160-169.

36. Bhave G, Karim F, Carlton SM, Gereau RW 4th. Peripheral group I metabotropic glutamate receptors modulate nociception in mice. Nat Neurosci. 2001;4(4):417-423.

37. Jackson DL, Graff CB, Richardson JD, Hargreaves 
KM. Glutamate participates in the peripheral modulation of thermal hyperalgesia in rats. Eur J Pharmacol. 1995;284(3):321-325.

38. Rudomin P. Primary afferent depolarization produced in Adelta and C fibres by glutamate spillover? New ways to look at old things. J Physiol. 2000;528 pt 1:1.

39. McNearney T, Speegle D, Lawand N, Lisse J, Westlund KN. Excitatory amino acid profiles of synovial fluid from patients with arthritis. J Rheumatol. 2000;27(3):739-745.

40. Hansen KB, Yuan H, Traynelis SF. Structural aspects of AMPA receptor activation, desensi- tization and deactivation. Curr Opin Neurobiol. 2007;17(3):281-288.

41. Lee CJ, Labrakakis C, Joseph DJ, Macdermott AB. Functional similarities and differences of AMPA and kainate receptors expressed by cultured rat sensory neurons. Neuroscience. 2004;129(1):35-48.

42. Cho CH, St-Gelais F, Zhang W, Tomita S, Howe JR Two families of TARP isoforms that have distinct effects on the kinetic properties of AMPA receptors and synaptic currents. Neuron. 2007;55(6):890-904.

43. Fukaya M, Watanabe M. Improved immunohistochemical detection of postsynaptically located PSD-95/SAP90 protein family by protease section pretreatment: a study in the adult mouse brain. J Comp Neurol. 2000;426(4):572-586.

44. Moser M, et al. Kindlin-3 is required for beta 2 integrin-mediated leukocyte adhesion to endothelial cells. Nat Med. 2009;15(3):300-305.

45. Schmidtko A, et al. Essential role of the synaptic vesicle protein synapsin II in formalin-induced hyperalgesia and glutamate release in the spinal cord. Pain. 2005;115(1-2):171-181.

46. Luo C, Seeburg PH, Sprengel R, Kuner R. Activitydependent potentiation of calcium signals in spinal sensory networks in inflammatory pain states. Pain. 2008;140(2):358-367. 